\title{
Fecho integral de ideais analíticos e equisingularidade
}

\author{
Claudia Rebouças Lima Fernandes
}

Orientador: Prof. Dr. Marcelo José Saia

Dissertação apresentada ao Instituto de Ciências Matemáticas e de Computação - ICMC-USP, como parte dos requisitos para obtenção do título de Mestre em Matemática.

USP - São Carlos

Novembro/2001 
A Comissĩa Julgadora:

Prof. Dr. Marcelo José Saia

Profa. Dra. Maria Aparecida Soares Ruas

Profa. Dra. Neuza Kazuko Kakuta 
Aos meus pais,

José e Raimunda. 


\title{
Agradecimentos
}

\author{
A Deus pelas bênçãos recebidas.
}

À minha família, em especial aos meus pais, pela dedicação e compreensão que foram absolutamente necessárias para a concretização deste sonho.

Ao meu esposo e colega de profissão, Alexandre, pelo companherismo expressado na paciência e dedicação que teve nas inúmeras vezes em que me deu suporte matemático que não possuía e que foi fundamental para a realização deste trabalho.

Ao Prof. Dr. Marcelo Saia pelo voto de confiança, trabalho de correção e, principalmente, pela escolha do tema.

Ao ICMC e ao Grupo de Singularidades pelo excelente suporte técnico dado.

A todos que direta ou indiretamente contribuíram para a realização deste. 


\section{Resumo}

Motivados por um resultado de B. Teissier que caracteriza algebricamente a Whitney equisingularidade de hipersuperfícies complexas em termos do fecho integral de um certo ideal, estudamos nesse trabalho um teorema, também devido a $\mathrm{B}$. Teissier, no qual são apresentadas condições equivalentes para um elemento pertencer ao fecho integral de um ideal. Utilizando esse teorema, fazemos um estudo no qual relacionamos o fecho integral, poliedro de Newton e multiplicidade de ideais Newton não-degenerados. Finalmente, aplicamos os resultados sobre fecho integral de ideais para a questão da equisingularidade de Whitney de famílias de hipersuperfícies complexas e a trivialidade topológica de famílias de germes de funções. 


\section{Abstract}

Motivated for a result of $\mathrm{B}$. Teissier which characterizes algebrically the Whitney equisingularity of complex hypersurfaces in terms of the integral closure of a certain ideal, we study in this work a theorem, also due the B. Teissier, in which are presented conditions equivalents for an element to belong to the integral closure of an ideal. Using this theorem, we make a study in which we relate the integral closure, polyhedron of Newton and multiplicity of Newton non-degenerate ideals. Finally, we apply the results on integral closure of ideals to the question of Whitney equisingularity of families of complex hypersurfaces and the topological triviality of families of germs of functions. 


\section{Sumário}

$\begin{array}{ll}\text { Introdução } & 3\end{array}$

1 Preliminares 6

1.1 Álgebra comutativa . . . . . . . . . . . . . . . . 6

1.1.1 Função de ordem e álgebras graduadas . . . . . . . . . . 7

1.2 Geometria analítica . . . . . . . . . . . . . . . 9 9

1.2.1 Funções holomorfas e variedades analíticas . . . . . . . . . 9

$1.2 .2 \mathrm{O}$ anel $\mathcal{O}_{X} \ldots \ldots \ldots \ldots 11 \ldots \ldots \ldots$

1.2.3 Funções meromorfas .................. 14

1.2 .4 Normalização . . . . . . . . . . . . . . 14

1.2 .5 Explosão (Blowing-up) ................... 15

1.2.6 Resolução de singularidades . . . . . . . . . . . . 16

1.3 Singularidades . . . . . . . . . . . . . . 16

1.3.1 Germes de funções . . . . . . . . . . . . . . 16

2 Fecho integral de ideais analíticos $\quad 18$

2.1 Fecho integral de ideais . . . . . . . . . . . . . 18

2.2 A Função de ordem $\bar{v}_{I} \ldots \ldots \ldots \ldots \ldots$

2.3 Ideais em $\mathcal{O}_{X} \ldots \ldots \ldots \ldots \ldots \ldots$

3 Filtração de Newton e não-degeneração $\quad 39$

3.1 A filtração de Newton . . . . . . . . . . . . . . . . . . 39

3.2 Ideais monomiais . . . . . . . . . . . . . . . . . 43 
3.3 Caracterizando ideais Newton não-degenerados . . . . . . . . . . . 50

4 Equisingularidade $\quad 57$

4.1 Whitney equisingularidade . . . . . . . . . . . 57

4.1.1 Whitney equisingularidade e o fecho integral de ideais . . . . . 58

4.2 Trivialidade topológica . . . . . . . . . . . . . 60

4.2.1 Trivialidade topológica e fecho integral . . . . . . . . . 61

4.2.2 Trivialidade topológica e poliedro de Newton . . . . . . . . . 62

$\begin{array}{ll}\text { Bibliografia } & 65\end{array}$ 


\section{Introdução}

O problema de classificação de familias de germes de funções (ou aplicações ) é um dos principais tópicos de pesquisa em Teoria de Singularidades e os trabalhos de Teissier em [20] e [22], Yoshinaga em [24], Greuel em [6] e Gaffney mostram que as relações de dependência integral de ideais e módulos são muito importantes na descrição infinitesimal de questões envolvendo a Whitney equisingularidade de variedades e hipersuperfícies e a trivialidade topológica de familias de germes de funções e aplicações .

Em [22], Teissier caracteriza algebricamente a Whitney equisingularidade de famílias de hipersuperfícies complexas em termos do fecho integral de um ideal analítico dependente dessa família. É devida ao próprio Teissier a efetividade desse resultado; a partir de idéias de H. Hironaka, ele formula um teorema de caracterização (completa) do fecho integral de ideais analíticos (Teorema 2.3.6). Por outro lado, como podemos ver em [14] e [15], a construção de poliedros de Newton associados a conjuntos de germes de função permite a determinação do fecho integral de ideais e conseqüentemente a determinação de condições para a preservação do tipo de singularidade de famílias, tais como trivialidade topológica e Whitney equisingularidade.

Neste trabalho expomos o resultado de caracterização de fecho integral citado e apresentamos aplicações significativas. Dividimos este trabalho da seguinte forma.

No capítulo 1, encontram-se os pré-requisitos: definições e resultados básicos de álgebra comutativa; algumas noções de geometria analítica como: variedade analítica, funções holomorfas e meromorfas, normalização, explosão e resolução de singularidades de variedade. Consta ainda nessa lista de pré-requisitos definições de Teoria de Singularidades. 
No capítulo 2, realizamos um estudo de fecho integral de ideais e apresentamos o Teorema de Caracterização.

No capítulo 3, apresentamos interessantes aplicações do Teorema de Caracterização. Relacionamos multiplicidade, poliedro de Newton e fecho integral de ideais Newton não-degenerados.

Finalmente no capítulo 4, abordamos a questão da equisingularidade. Como aplicação do cálculo de fecho integral de ideais a partir do poliedro de Newton, são dadas condições necessárias e suficientes para a Whitney equisingularidade de famílias de germes de hipersuperfícies Newton não-degenerados, bem como para a trivialidade topológica de famílias de germes de funções Newton não-degenerados. 


\section{Lista de Notações}

$\mathbb{Z}$ anel dos inteiros

$\mathbb{N}$ conjunto dos inteiros não negativos

$\mathbb{R}$ corpo dos números reais

$\overline{\mathbb{R}}=\mathbb{R} \cup\{\infty\}$

$\mathbb{R}_{0}$ reais não negativos

$\overline{\mathbb{R}_{0}}=\mathbb{R}_{0} \cup\{\infty\}$

$\mathbb{C}$ corpo dos números complexos

$\mathbb{C}\left\{x_{1}, \ldots, x_{n}\right\}$ anel das séries de potências convergentes na origem 


\section{Capítulo 1}

\section{Preliminares}

\section{1 Álgebra comutativa}

Os resultados e definições desta seção são parte do livro [12].

Entendemos por anel um anel comutativo com unidade $1 \neq 0$. Um anel $A$ é noetheriano quando todos os seus ideais são finitamente gerados, ou equivalentemente, toda cadeia ascendente

$$
I_{1} \subset I_{2} \subset I_{3} \subset \cdots
$$

de ideais de $A$ é estacionária; isto é, existe $n_{0} \in \mathbb{N}$ tal que $I_{n_{0}}=I_{n_{0}+k}, \quad \forall \quad k \in \mathbb{N}$.

Exemplo 1.1.1. $A=\mathbb{Z}, A=K$ com $K$ um corpo.

O seguinte resultado devido a D. Hilbert nos permite ampliar a lista de exemplos.

Teorema 1.1.2 (Teorema da Base de Hilbert). Se A é um anel noetheriano, então $A[X]$ é noetheriano.

Exemplo 1.1.3. Com esse teorema, obtemos que $\mathbb{Z}[X], \mathbb{Z}\left[X_{1}, X_{2}\right], \mathbb{Z}\left[X_{1}, X_{2}, \ldots, X_{n}\right]$, $K[X], K\left[X_{1}, X_{2}\right], K\left[X_{1}, X_{2}, \ldots, X_{n}\right]$ são anéis noetherianos.

Um anel $A$ que possui apenas um ideal maximal é chamado um anel local. Expressamos o fato de $A$ ser um anel local com ideal maximal $\eta$ através da notação $(A, \eta)$. Nesse caso, o corpo $k=\frac{A}{\eta}$ é chamado o corpo residual de $A$ e usamos a notação 
$(A, \eta, k)$. Se $A$ é um anel local, então os elementos de $A$ não pertencentes a $\eta$ são invertíveis.

Definição 1.1.4. Seja $A$ um anel. Uma cadeia de ideais primos de comprimento $l$ em $A$, é uma seqüência ascendente $\mathcal{P}_{0} \varsubsetneqq \mathcal{P}_{1} \varsubsetneqq \cdots \varsubsetneqq \mathcal{P}_{l} \varsubsetneqq A$ de ideais primos. $A$ dimensão de Krull de A é o máximo dos comprimentos das cadeias de ideais primos em $A$. Denotamos essa dimensão por $\operatorname{dim}(A)$.

Sejam $(A, \eta, k)$ um anel local e $I$ um ideal de $A$. Então, $\frac{A}{I}$ tem estrutura de espaço

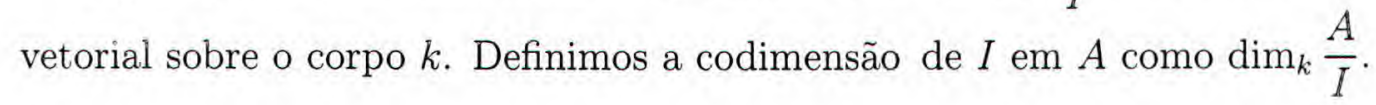

Definição 1.1.5. Sejam $(A, \eta, k)$ um anel noetheriano local e $I$ um ideal de codimensão finita em A. A multiplicidade de I é definida como

$$
e(I)=\lim _{n \rightarrow \infty} \frac{d !}{n^{d}} \operatorname{dim}_{k} \frac{A}{I^{n}} \quad \text { em que } \quad d=\operatorname{dim}(A) .
$$

Um ideal $I$ do anel local $(A, \eta, k)$ é primário se, dados $a b \in I$ então ou $b \in$ $I$ ou $a^{m} \in I$ para algum $m$. O ideal $I$ é $\eta$-primário quando é primário e, $\sqrt{I}=\eta$, com $\sqrt{I}$ denotando oradical de $I$.

Teorema 1.1.6. Sejam $(A, \eta, k)$ um anel noetheriano local e I, $J$ ideais de codimensão finita em A. Então,

(i) $e\left(I^{n}\right)=n^{d} e(I)$

(ii) Se I e J são ambos ideais $\eta$-primários e $I \supset J$, então $e(I) \leq e(J)$.

\subsubsection{Função de ordem e álgebras graduadas}

Definição 1.1.7. Sejam $A$ um anel e $\mu: A \rightarrow \overline{\mathbb{R}_{0}}$ uma aplicação. Dizemos que $\mu$ é uma função de ordem quando satisfaz as seguintes condições:

(i) $\mu(x+y) \geq \inf \{\mu(x), \mu(y)\}$

(ii) $\mu(x y) \geq \mu(x)+\mu(y)$

(iii) $\mu(0)=\infty, \mu(1)=0$. 
Quando vale a igualdade em (ii) para quaisquer $x, y \in A, \mu$ é dita uma valoração.

Definição 1.1.8. Seja $A$ um anel. Uma filtração (decrescente) sobre $A$ é uma seqüência decrescente $\left(A_{d}\right)_{d \in \mathbb{R}_{0}}$ de subgrupos de $A$, ou seja, $A_{d} \supset A_{d+k}$ para $d, k \in \mathbb{R}_{0}$ verificando:

(i) $A_{d} A_{e} \subset A_{d+e}$ para d,e $\in \mathbb{R}_{0}$

(ii) $A_{0}=A$

(iii) Existe $d \in \mathbb{R}_{+}$tal que $A_{d} \neq A$.

Neste caso, dizemos que $A$ é um anel filtrado pela seqüência $\left(A_{d}\right)_{d \in \mathbb{R}_{0}}$.

Observação 1.1.9. Se $\mu$ é uma função de ordem, então $\mu(-x)=\mu(x)$. Além disso, se $\mu(x)<\mu(y)$, então $\mu(x+y)=\mu(x)$. Se $A$ é um anel filtrado, então $A_{d}$ é um ideal próprio de $A$ para todo $d \in \mathbb{R}_{+}$.

Observação 1.1.10. As noções de filtração e função de ordem são equivalentes. Ou seja, dada uma função de ordem $\mu$, consideramos $A_{d}=\{x \in A: \mu(x) \geq d\}, d \in \mathbb{R}_{0}$. Por outro lado, dada uma filtração $\left(A_{d}\right)_{d \in \mathbb{R}_{0}}$ em $A$, definimos a função de ordem $\mu(x)=\sup \left\{d: x \in A_{d}\right\}$.

Definição 1.1.11. Dados $A, G$ anéis, $G$ é uma $A$-álgebra se existe um homomorfismo $f: A \rightarrow G$.

Definição 1.1.12. Sejam $A$ um anel e $G$ uma A-álgebra. $G$ é uma A-álgebra graduada se possui uma decomposição

$$
G=\oplus_{d \in \mathbb{R}_{0}} G_{d}
$$

com

(i) $G_{d}$ é um A-módulo para $d \in \mathbb{R}_{0}$

(ii) $G_{0}=A$

(iii) $G_{d_{1}} G_{d_{2}} \subset G_{d_{1}+d_{2}}$ para $d_{1}, d_{2} \in \mathbb{R}_{0}$. 
Sejam $A$ um anel e $\mu$ uma função de ordem. Considerando $A_{d}=\{x \in A: \mu(x) \geq$ $d\}$, e $A_{d}^{+}=\{x \in A: \mu(x)>d\}, \quad$ para $d \in \mathbb{R}_{0}$, então

$$
G r A=\oplus_{d \in \mathbb{R}_{0}} \frac{A_{d}}{A_{d}^{+}}
$$

é uma $\frac{A}{A_{0}^{+}}$-álgebra graduada.

Um elemento $x \in A$ é homogêneo, ou uma forma, de grau $i$ se $x \in A_{i}$. Além disso, dado $x \in A$ escrevemos de forma única $x=\sum_{i \in \mathbb{R}_{0}} x_{i}$ em que $x_{i} \neq 0$ apenas para uma quantidade finita de índices $i$. O termo $x_{i}$ é a componente de grau $i$ de $x$.

\subsection{Geometria analítica}

\subsubsection{Funções holomorfas e variedades analíticas}

Dado $H \subset \mathbb{C}^{m}$ aberto, uma função $f: H \rightarrow \mathbb{C}$ é holomorfa se, e somente se, cada derivada $\frac{\partial f}{\partial x_{i}}$ é contínua em $H$. Se $Q$ é um subconjunto qualquer de $\mathbb{C}^{m}$, então $g: Q \rightarrow \mathbb{C}$ é holomorfa se, e somente se, existe um aberto $H$ de $\mathbb{C}^{m}$ tal que $Q \subset H, g$ está definida e é holomorfa em $H$. Uma aplicação $f: H \rightarrow \mathbb{C}^{n}$ é holomorfa se, e somente se, cada função coordenada $f_{i}$ o é.

Um subconjunto $V$ de $\mathbb{C}^{n}$ é dito analítico próximo ao ponto $p$, ou é uma variedade perto de $p$, se, e somente se, existem uma vizinhança $U$ de $p$ e funções holomorfas $f_{1}, f_{2}, \ldots, f_{s}$ tais que $V \cap U=Z\left(\left\{f_{1}, f_{2}, \ldots, f_{s}\right\}\right)=\left\{q: f_{1}(q)=f_{2}(q)=\ldots=\right.$ $\left.f_{s}(q)=0\right\}$. Assim, $V$ é analítico nas proximidades dos pontos de $V \cap U$.

Definição 1.2.1. Um subconjunto $V$ de $\mathbb{C}^{n}$ é localmente analítico, ou é localmente uma variedade, se é analítico nas proximidades de seus pontos.

Dados um aberto $H$ em $\mathbb{C}^{n}$ e $V \subset H \subset \mathbb{C}^{n}$ um subconjunto, dizemos que $V$ é analítico em $H$, ou é uma variedade em $H$, se, e somente se, $V$ é analítico próximo a cada um dos pontos de $H$.

Seja $V$ um espaço Hausdorff. Um sistema local de coordenadas analíticas em $V$ é definido como um homeomorfismo $\mathcal{X}$ de uma variedade local $X$ em $\mathbb{C}^{n}$, para algum 
$n$, em um subconjunto aberto de $V$. Se $p \in \mathcal{X}(X)$, então temos um sistema de coordenadas em $p \in V$.

Definição 1.2.2. Um espaço añlítico com sistemas de coordenadas é um espaço Hausdorff $V$ com um conjunto $\Im$ de sistemas de coordenadas analiticas em $V$, tais que

(i) as imagens de um subconjunto finito ou enumerável de sistemas de coordenadas cobrem $V$,

(ii) Se $\mathcal{X}_{i}$ e $\mathcal{X}_{j}$ são sistemas de coordenadas tais que a interseção de suas imagens é não vazia, então $\mathcal{X}_{j}^{-1} \circ \mathcal{X}_{i}$ é um bi-holomorfismo.

Definição 1.2.3. Um espaço analítico é um espaço Hausdorff $V$ com um conjunto $\Im$ de sistemas de coordenadas analíticas, para o qual as condições (i) e (ii) da definição anterior são satisfeitas e:

se $\mathcal{X}$ é um sistema de coordenadas analítico qualquer em $V$, tal que para cada $\mathcal{X}_{k} \in$ $\Im, \quad \mathcal{X}_{k}^{-1} \circ \mathcal{X}$ é um bi-holomorfismo, então $\mathcal{X} \in \Im$.

Definição 1.2.4. Sejam $V \subset \mathbb{C}^{n}$ uma variedade local e $f: V \rightarrow \mathbb{C}$ uma função. Então, $f$ é holomorfa se, para cada $p \in V$, existem uma vizinhança $U \subset \mathbb{C}^{n}$ de $p e$ uma função holomorfa $f^{\prime}$ definida em $U$ tal que $f=f_{\mid V \cap U}^{\prime}$.

Definição 1.2.5. Um ponto $p$ de uma variedade local $V$ é um ponto regular de $V$ se, e somente se, $V$ é uma variedade analítica em uma vizinhança de $p$. Caso contrário, $p$ é um ponto singular. Denotamos o conjunto dos pontos regulares de $V$ por $V^{-} e, o$ conjunto dos pontos singulares por $\operatorname{Sing}(V)$.

Seja uma variedade local $V \subset \mathbb{C}^{n}$. A aplicação $f: V \rightarrow \mathbb{C}^{m}$ é holomorfa se cada $p \in V$ possui uma vizinhança $U$ na qual $f$ tem uma extensão holomorfa.

Observação 1.2.6. Seja $W$ uma variedade local em $\mathbb{C}^{m}$. Então, $F: V \rightarrow W$ é holomorfa se, e somente se, $f: V \rightarrow \mathbb{C}^{m}$ o é.

Definição 1.2.7. O espaço analítico $V$ é uma variedade analítica se, cada $p \in V$ está em um sistema de coordenadas cujo domínio é um subconjunto aberto de $\mathbb{C}^{r}$ para algum $r$. Nesse caso, $r$ é a dimensão de $V$. 
Dados $V$ um espaço analítico e $V_{1}$ um subconjunto aberto de $V$, temos que $f$ : $V_{1} \rightarrow \mathbb{C}$ é holomorfa se, para cada sistema de coordenadas $\mathcal{X}$ cuja imagem intersecta $V_{1}, f \circ \mathcal{X}$ é holomorfa em seu domínio.

O conjunto $\mathcal{O}(U)=\{f: U \rightarrow \mathbb{C}\}$ é um anel para todo conjunto aberto $U$.

\subsection{2 $\mathrm{O}$ anel $\mathcal{O}_{X}$}

Antes de definir o anel $\mathcal{O}_{X}$, precisamos introduzir a noção de feixe.

Seja $X$ um espaço topológico. Um feixe $\mathcal{F}$ de anéis [ respectivamente, módulos] sobre $X$, consiste do seguinte:

(i) Para cada aberto $U$ de $X$, existe um anel [módulo] $\Gamma(U, \mathcal{F})$ chamado conjunto de seções de $\mathcal{F}$ sobre $U$.

(ii) Para cada par de abertos $V \subseteq U$ de $X$, existe uma aplicação de restrição $r_{U, V}: \Gamma(U, \mathcal{F}) \rightarrow \Gamma(V, \mathcal{F}), r_{U, V}(s)=s_{\left.\right|_{V}}$ satisfazendo:

(a) $r_{U, U}=i d_{U}$ para cada aberto $U$ de $X$;

(b) $r_{V, W} \circ r_{U, V}=r_{U, W}$ para cada $W \subseteq V \subseteq U$ abertos de $X$.

(iii) Para cada aberto $U$ de $X$ e cada cobertura de $U$ por abertos $U=\cup_{i \in I} U_{i}$, de $X$ tem-se :

(a) para quaisquer $s_{1}, s_{2} \in \Gamma(U, \mathcal{F})$, se $s_{\left.\right|_{U_{i}}}=s_{\left.2\right|_{U_{i}}} \quad \forall i \in I$, então $s_{1}=s_{2}$;

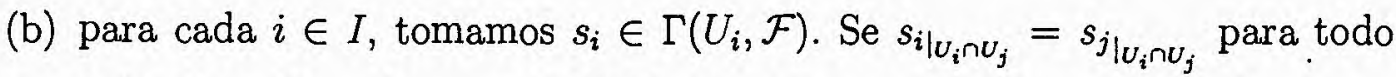
$i, j \in I$, então existe $s \in \Gamma(U, \mathcal{F})$ tal que $s_{l_{i}}=s_{i}$ para todo $i \in I$.

Exemplo 1.2.8. Dado um espaço topológico $X$, o feixe de funções contínuas, denotado por $\mathcal{C}^{0}$, é definido por

$$
\Gamma\left(U, \mathcal{C}^{0}\right):=\{f: U \rightarrow \mathbb{C}: f \text { é contínua }\}
$$

com a restrição natural.

Definição 1.2.9. Um feixe $\mathcal{F}$ é um feixe de $\mathbb{C}$-álgebras $s e \Gamma(U, \mathcal{F})$ é uma $\mathbb{C}$-álgebra, para toda aberto $U$ de $X$. 
Exemplo 1.2.10 (O feixe $\mathcal{O}_{X}$ ). Seja $X$ um espaço analítico. Para cada aberto $U \subset X$, definimos o anel

$$
\Gamma(U, \mathcal{F})=\{f: U \rightarrow \mathbb{C}: f \quad \text { é holomorfa }\} .
$$

Considerando as restrições naturais, temos um feixe de anéis $\mathcal{F}$. Este feixe é comumente chamado de feixe de funções holomorfas e denotado por $\mathcal{O}_{X}$. As seções de $\mathcal{O}_{X}$ sobre um aberto $U$, costumam ser denotadas por $\mathcal{O}_{X}(U)=\Gamma\left(U, \mathcal{O}_{X}\right)$. Sendo estas seções $\mathbb{C}$-álgebras, este feixe é um exemplo de feixe de $\mathbb{C}$-álgebra.

Definição 1.2.11. Seja $\mathcal{F}$ um feixe de anéis [módulos] sobre $X$. Definimos o talo (stalk) de $\mathcal{F}$ em $x$ como o anel [módulo]

$$
\mathcal{F}_{x}:=\{(U, s): U \text { é aberto de } \quad X, \quad x \in U, \quad \text { e } \quad s \in \Gamma(U, \mathcal{F})\}
$$

módulo a relação :

$(U, s) \sim(V, t) \Leftrightarrow \exists W$ aberto de $X$ contido em $U \cap V$ tal que $s_{\left.\right|_{W}}=t_{\left.\right|_{W}}$.

Exemplo 1.2.12. No feixe $\mathcal{O}_{X}$ sobre um espaço analítico $X$, o talo desse feixe no ponto $x$ é o anel $\mathcal{O}_{X, x}=\{f:(X, x) \rightarrow(\mathbb{C}, y)\}$.

Seja $X$ um espaço topológico. Dados dois feixes $\mathcal{F}$ e $\mathcal{G}$ em $X, \mathcal{F}$ é chamado um sub-feixe de $\mathcal{G}$ se para todo subconjunto aberto $V$ de $X, \Gamma(V, \mathcal{F}) \subset \Gamma(V, \mathcal{G})$ e a restrição em $\mathcal{F}$ coincide com a restrição em $\mathcal{G}$.

Um morfismo $\alpha: \mathcal{F} \rightarrow \mathcal{G}$ entre dois feixes de $X$ é uma coleção de aplicações

$$
\alpha(V): \Gamma(V, \mathcal{F}) \rightarrow \Gamma(V, \mathcal{G})
$$

para cada $V$ aberto de $X$ tal que o diagrama

$$
\begin{array}{lcc}
\Gamma(V, \mathcal{F}) & \stackrel{\alpha(V)}{\longrightarrow} & \Gamma(V, \mathcal{G}) \\
r_{V, U} \downarrow & & \downarrow \tilde{r}_{V, U} \\
\Gamma(U, \mathcal{F}) & \stackrel{\alpha(U)}{\longrightarrow} & \Gamma(U, \mathcal{G})
\end{array}
$$

comuta sempre que $U \subset V$ são abertos de $X$.

Um morfismo $\alpha: \mathcal{F} \rightarrow \mathcal{G}$ induz um homomorfismo

$$
\alpha_{x}: \mathcal{F}_{x} \rightarrow \mathcal{G}_{x}
$$


para todo $x \in X$ naturalmente.

Definição 1.2.13. O morfismo $\alpha$ é injetor [sobrejetor] se para todo $x \in X, \alpha_{x}$ é injetor [sobrejetor].

Dados um feixe $\mathcal{F}$ sobre o espaço topológico $X$ e um aberto $U$ de $X$, podemos definir um feixe em $U$ a partir de $\mathcal{F}$. Para cada aberto $V$ de $U$, definimos $\Gamma_{U}(V, \mathcal{F})=$ $\Gamma(V, \mathcal{F})$. As aplicações de restrição são as mesmas. Esse feixe é chamado de restrição do feixe $\mathcal{F}$ em $U$ e denotado por $\mathcal{F}_{\text {lU }}$.

Dado um feixe $\mathcal{F}$ sobre um espaço topológico $X$, chamamos de seção global à seção $\Gamma(X, \mathcal{F})$. Por abuso de linguagem, podemos nos referir a $\Gamma\left(U, \mathcal{F}_{U}\right)$ como a seção global de $\mathcal{F}$ em $U$, em que $U$ é um aberto de $X$ e $\mathcal{F}_{U}$ é o feixe $\mathcal{F}$ restrito a $U$.

\section{Feixe de ideais}

Sejam $X$ um espaço analítico e $Y$ um subespaço de $X$. Temos então, a aplicação inclusão

$$
i: Y \hookrightarrow X
$$

Associado a $i$, temos um morfismo de feixes $i^{*}: \mathcal{O}_{X} \rightarrow \mathcal{O}_{Y}$ tal que para cada aberto $U$ de $X$,

$$
\begin{aligned}
i^{*}: \mathcal{O}_{X}(U) & \rightarrow \mathcal{O}_{Y}(U \cap Y) \\
f & \mapsto f \circ i .
\end{aligned}
$$

Denotamos $\mathcal{I}=\operatorname{ker}\left(i^{*}\right)$. Então, $\mathcal{I}$ é dito o feixe de ideais sobre $X$ que define $Y$.

Definição 1.2.14. Seja $\mathcal{F}$ um feixe de anéis em um espaço topológico $X$. Um $\mathcal{F}$ módulo $\mathcal{M}$ é coerente se localmente tem uma representação

$$
\bigoplus_{i \in I} \mathcal{F}_{i} \rightarrow \bigoplus_{j \in J} \mathcal{F}_{j} \rightarrow \mathcal{M}_{l u} \rightarrow 0
$$

em que $\mathcal{F}_{i}=\mathcal{F}_{j}=\mathcal{F}_{\mid U} \quad \forall i \in I, j \in J \quad$ e $\quad I, J$ são conjuntos finitos.

Um feixe de ideais $\mathcal{I}$ no feixe de anéis $\mathcal{F}$ é um $\mathcal{F}$-módulo. Assim, $\mathcal{I}$ é um $\mathcal{F}$-ideal coerente quando é um $\mathcal{F}$-módulo coerente. 
Definição 1.2.15. Seja $A$ um anel. Um ideal I de $A$ é invertível, quando é principal e pode ser gerado por um elemento $g \in A$ não divisor de zero, isto é, $I=g A$.

Temos uma definição análoga para um feixe de ideais no feixe de funções regulares $\mathcal{O}_{X}$

Um feixe de ideais $\mathcal{I}$ em $\mathcal{O}_{X}$ é invertível se, e somente se, $\mathcal{I}$ é localmente principal e gerado por um elemento não divisor de zero. Ou seja, dado $x \in X$, existe uma vizinhança $V$ de $x$ em $X$ tal que o ideal $\mathcal{I}(V)$ é invertível no anel $\mathcal{O}_{X}(V)$. Ou ainda, dado $x \in X$, existem uma vizinhança $V$ de $x$ e uma função holomorfa $g: V \rightarrow \mathbb{C}$ que é não divisora de zero em $\mathcal{O}_{X}(V)$ e $\mathcal{I}(V)=g \mathcal{O}_{X}(V)$. Em particular, quando tomamos o germe do feixe $\mathcal{I}$ em $y \in V$, temos

$$
\mathcal{I}_{y}=g \mathcal{O}_{X, y}
$$

$\operatorname{com} \mathcal{O}_{X, y}=\left\{\frac{f}{h}: f \in \mathcal{O}_{X}, h(y) \neq 0\right\}$

\subsubsection{Funções meromorfas}

Uma função meromorfa em um espaço analítico $X$ consiste de um par $(H, f)$ em que $H$ é um subconjunto aberto e denso de $X$ e $f$ é uma função holomorfa em $H$ com a seguinte propriedade:

para cada $p \in X$, existem uma vizinhança $U$ de $p$ e funções holomorfas $\varphi$ e $\psi$ em $U$ tais que o conjunto dos zeros de $\psi$, denotado por $Z(\psi)$ não é denso em parte alguma de $U$ e $f=\frac{\varphi}{\psi}$ em $U \cap\left(H \backslash Z_{\psi}\right)$.

Observação 1.2.16. A função $f$ pode ser extendida a uma função holomorfa em um subconjunto aberto $H_{f}$ cujo complementar $N_{f}$ é uma subvariedade que não é densa em parte alguma de $X$. Ao extendermos, podemos subistituir o par $(H, f)$ pelo par $\left(H_{f}, f\right)$.

\subsubsection{Normalização}

Um espaço analítico $X$ é reduzido quando o feixe de anéis $\mathcal{O}_{X}$ é reduzido, isto é, não possui elementos nilpotentes. O espaço $X$ é normal em uma vizinhança de $x \in X$ 
se existe um aberto $U$ de $X$ contendo $x$ tal que $\mathcal{O}_{X}(U)$ é um anel normal.

Definição 1.2.17. Sejam $X$ e $Y$ espaços Hausdorff localmente compactos, e $f: A \rightarrow$ $Y$ uma aplicação contínua com $A \subset X$. Então, $f$ é própria em $Y$ se, e somente, se para cada subconjunto compacto $Q$ de $Y, 耳^{-1}(Q)$ é compacto.

Definição 1.2.18. Seja $X$ um espaço analítico. Uma tripla $(W, X, \pi)$ é uma normalização de $X$ se, e somente se as seguintes condições são satisfeitas:

(i) W é um espaço analítico normal,

(ii) $\pi: W \rightarrow X$ é holomorfa, própria, sobre $X$, e com fibras finitas,

(iii) $O$ conjunto $W^{\prime}=\pi^{-1}\left(X^{-}\right)$está contido em $W^{-}$e é denso em $W$,

(iv) $\pi_{\left.\right|_{w^{\prime}}}$ é um bi-holomorfismo em $X^{-}$.

Teorema 1.2.19 ( [23], pag. 256). Seja $X$ um espaço analítico. Então, existe uma normalização $(W, X, \pi)$ de $X$.

\subsubsection{Explosão (Blowing-up)}

Sejam $X$ um espaço analítico reduzido e $\mathcal{I}$ um feixe coerente de ideais sobre $X$. Seja $\Pi: \widetilde{X} \rightarrow X$ um morfismo próprio sobrejetivo. Suponhamos que para todo morfismo $f: Z \rightarrow X$, tal que $f^{-1}(\mathcal{I}) \mathcal{O}_{Z}$ é um feixe de ideais invertível em $Z$, exista um único morfismo $g: Z \rightarrow \widetilde{X}$ tal que $\Pi \circ g=f$. Nesse caso, $\Pi$ é dita uma explosão de $X$ ao longo de $\mathcal{I}$.

Podemos ver em ([7], pag. 163) que dados um espaço analítico reduzido $X$ e um feixe de ideais $\mathcal{I}$ sobre $X$, existe uma explosão ao longo de $\mathcal{I}$.

O seguinte resultado apresenta a propiedade de uma explosão que nos interessa.

Teorema 1.2.20. Sejam $X$ um espaço analítico reduzido, $\mathcal{I}$ um feixe coerente de ideais e $\Pi: \widetilde{X} \rightarrow X$ uma explosão de $X$ ao longo de $\mathcal{I}$. Então, a imagem inversa do feixe de ideais $\mathcal{I}, \widetilde{\mathcal{I}}=\Pi^{*}(\mathcal{I}) \mathcal{O}_{\tilde{X}}$ é um feixe de ideais invertivel sobre $\tilde{X}$. 


\subsubsection{Resolução de singularidades}

Seja $X$ um espaço analítico reduzido. Uma resolução de singularidades de $X$ 'é um morfismo

$$
\Phi: X^{\prime} \rightarrow X
$$

satisfazendo as seguintes propriedades:

(i) $\Phi$ é própria e sobrejetiva;

(ii) $X^{\prime}$ é não singular;

(iii) $\Phi_{\Phi_{\Phi^{-1}\left(X^{-}\right)}}$é um bi-holomorfismo sobre $X^{-}$.

Hironaka mostra em [8] que dado um espaço analítico reduzido $X$, existe uma resolução de singularidades de $X$.

\subsection{Singularidades}

\subsubsection{Germes de funções}

Sejam $X$ um espaço topológico e $a \in X$. Consideremos

$$
S=\{f: V \rightarrow \mathbb{C}: V \subset X \text { é vizinhança de } a\} .
$$

Dados $f, g \in S$, dizemos que $f$ é equivalente a $g$ em $a$ e, denotamos por $f \sim_{a} g$, se existe uma vizinhança $U$ de $a$ tal que $f_{\mid U}=g_{\mid U}$.

Observação 1.3.1. Esta relação é uma relação de equivalência.

Definição 1.3.2. Dados $X, S$ e a como acima, se $f \in S$, a classe de equivalência de $f$ sobre essa relação é o germe de $f$ em a; denotamos tal classe por $f:(X, a) \rightarrow(\mathbb{C}, y)$ em que $y=f(a)$.

O conjunto das classes de equivalência módulo a relação descrita acima, é um anel. Quando $X=\mathbb{C}^{n}$ e $S$ é o conjunto das funções holomorfas definidas em vizinhanças 
de $a$ tomando valores em $\mathbb{C}$, denotamos o conjunto dos germes em $a$ por $\mathcal{O}_{n}^{a}$. Este é o anel dos germes holomorfos em $a$. Quando $a=0$, escrevemos apenas $\mathcal{O}_{n}$.

Observamos que a aplicação

$$
\begin{aligned}
\mathcal{O}_{n}^{a} & \rightarrow \mathcal{O}_{n} \\
f & \mapsto f^{\prime} \quad \text { com } \quad f^{\prime}(x)=f(x+a)
\end{aligned}
$$

é um isormofismo. Assim, podemos nos preocupar apenas em estudar $\mathcal{O}_{n}$.

Lema 1.3.3. $O$ anel $\mathcal{O}_{n}$ é isomorfo ao anel das séries de potências convergentes na origem.

Lema 1.3.4 (ver [23]). A respeito de $\mathcal{O}_{n}$ valem as seguintes propriedades:

(i) $\mathcal{O}_{n}$ é isomorfo ao anel das séries de potências convergentes na origem.

(ii) $O$ germe $f \in \mathcal{O}_{n}$ é uma unidade nesse anel se, e somente se, $f(0) \neq 0$.

(iii) $\mathcal{O}_{n}$ é um domínio de fatoração única.

(iv) $\mathcal{O}_{n}$ é noetheriano.

Dados uma função de ordem $\mu$ em $\mathcal{O}_{n}$ e um germe $f=\sum_{s} a_{s} x_{1}^{s_{1}} \ldots x_{n}^{s_{n}}$ em $\mathcal{O}_{n}$, definimos a parte principal de $f$ com respeito a filtração determinada por $\mu$, denotada por in $(f)$, como

$$
\operatorname{in}(f)=\sum_{\mu\left(a_{k} x_{1}^{k_{1}} \ldots x_{n}^{k_{n}}\right)=\mu(f)} a_{k} x_{1}^{k_{1}} \ldots x_{n}^{k_{n}}
$$




\section{Capítulo 2}

\section{Fecho integral de ideais analíticos}

Os resultados descritos neste capítulo encontram-se em [18].

\subsection{Fecho integral de ideais}

Dados $R$ um anel comutativo com unidade 1 e $I$ um ideal próprio de $R$, associado a $I$ temos uma função de ordem natural, a saber,

$$
v_{I}(x)=\sup \left\{m \in \mathbb{N}: x \in I^{m}\right\}
$$

Observamos que

$$
x \in I \quad \text { se, e somente se, } \quad v_{I}(x) \geq 1 .
$$

Em $R$, definimos o conjunto constituído dos elementos que satisfazem uma equação do tipo

$$
x^{m}+a_{1} x^{m-1}+\ldots+a_{m}=0 \quad \text { com } \quad a_{i} \in I^{i} \quad \text { ou equivalentemente, } \quad v_{I}\left(a_{i}\right) \geq i .
$$

Tal conjunto é chamado o fecho integral de $I$ e denotado por $\bar{I}$.

Em geral, não é uma tarefa simples obter os elementos de $\bar{I}$. Descrevemos neste capítulo, vários resultados que nos permitem a obtenção dos elementos de $\bar{I}$. Em especial, construímos uma função de ordem $\mu$ que depende de $I$ e tal que, no caso analítico,

$$
x \in \bar{I} \quad \text { se, e somente se, } \quad \mu(x) \geq 1 .
$$


Antes de construir tal função, vejamos alguns resultados a respeito do fecho integral de um ideal.

Dados $A \subset B$ anéis e $f \in B, f$ é inteiro sobre $A$ se satisfaz a uma equação da forma

$$
f^{m}+a_{1} f^{m-1}+\ldots+a_{m}=0 \quad \text { com } \quad a_{i} \in A .
$$

Seja $R[T]$ o anel de polinômios a uma variável $T$ sobre $R$. Denotamos por $\mathcal{P}(I)$ o subanel $\bigoplus_{n \in \mathbb{N}} I^{n} T^{n}$ de $R[T]$. O lema abaixo, relaciona a noção de dependência inteira sobre um ideal com a noção de dependência inteira sobre um anel.

Lema 2.1.1. Seja $f \in R$. Então, $f \in \bar{I}$ se, e somente se, o elemento $f T$ é inteiro sobre o anel $\mathcal{P}(I)$ no sentido usual.

Demonstração: Seja $f^{k}+a_{1} f^{k-1}+\ldots+a_{k}=0$ uma relação de dependência inteira de $f$ sobre $I$. Então, $(f T)^{k}+a_{1} T(f T)^{k-1}+\ldots+a_{k} T^{k}$ é uma relação de dependência inteira de $f T$ sobre $\mathcal{P}(I)$. Reciprocamente, dada uma relação de dependência inteira

$$
(f T)^{k}+b_{1}(f T)^{k-1}+\ldots+b_{k}=0
$$

de $f T$ sobre $\mathcal{P}(I)$, para cada $i \in\{1, \ldots, k\}$ temos $b_{i}=b_{i 0}+b_{i 1} T+\ldots+b_{i n_{i}} T^{n_{i}}$ com $b_{i j} \in I^{j}$. Substituindo essas expressões em (2.2) e agrupando os termos segundo as potências de $T$, obtemos que o coeficiente de $T^{j}$ é igual a zero, para $j \in\{1, \ldots, k\}$. Em particular, $0=$ coeficiente $\left(T^{k}\right)=f^{k}+b_{11} f^{k-1}+b_{22} f^{k-2}+\ldots+b_{k k}$, que é uma relação de dependência inteira de $f$ sobre $I$.

Corolário 2.1.2. O fecho integral de um ideal $I, \bar{I}=\{f \in R: f$ é inteiro sobre $I\}$ é um ideal de R. $O$ ideal $I$ é integralmente fechado quando $I=\bar{I}$.

Demonstração: De fato, se $f, g \in \bar{I}$, então $f T, g T$ são inteiros sobre $\mathcal{P}(I)$. Como $\overline{\mathcal{P}(I)}$ é um subanel, $(f+g) T=f T+g T$ é inteiro sobre $\mathcal{P}(I)$. Logo, $f+g \in \bar{I}$. Além disso, dados $f \in \bar{I}, g \in R$ e uma relação de dependência inteira de $f$ sobre $I$, $f^{k}+a_{1} f^{k-1}+\ldots+a_{k}=0$, a expressão $(g f)^{k}+a_{1} g(g f)^{k-1}+\ldots+a_{k} g^{k}=0$ é uma relação de depedência inteira de $g f$ sobre $I$.

Observação 2.1.3. Se $I$ e $J$ são ideais em $R \operatorname{com} I \subset J$, então $\bar{I} \subset \bar{J}$. 
Lema 2.1.4. $I \subset \bar{I} \subset \sqrt{I}$. Em particular, se $I=\sqrt{I}$, então $I$ é integralmente fechado.

Demonstração: Se $f \in \bar{I}$ e $f^{k}+a_{1} f^{k-1}+\ldots+a_{k}=0$ é uma relação de dependência inteira de $f$ sobre $I$, então segue de $a_{j} \in I^{j}$ que $a_{j} f^{k-j} \in I^{j} \subset I$ para $j \in\{1, \ldots, k\}$. Assim, $f^{k}=-a_{k}-a_{k-1} f-\ldots-a_{1} f^{k-1} \in I$ e, portanto, $f \in \sqrt{I}$. Daí, $I \subset \bar{I} \subset \sqrt{I}$.

Lema 2.1.5. O fecho integral de $\mathcal{P}(I)=\bigoplus_{n \in \mathbb{N}} I^{n} T^{n}$ em $R[T]$ é $\bigoplus_{n \in \mathbb{N}} \overline{I^{n}} T^{n}$.

Demonstração: $\mathrm{O}$ fecho integral de $\mathcal{P}(I)$ em $R[T]$ é um subanel graduado $\overline{\mathcal{P}(I)}=$ $\bigoplus_{n \in \mathbb{N}} J_{n} T^{n}$ de $R[T]$ [ver [2], pag. 321]. Então, é suficiente determinar as componentes homogêneas, ou seja, devemos mostrar que $J_{n}=\overline{I^{n}}$.

Dado $f \in J_{n}, f T^{n}$ é inteiro sobre $\mathcal{P}(I)$. Então, existe uma relação $\left(f T^{n}\right)^{k}+$ $b_{1}\left(f T^{n}\right)^{k-1}+\ldots+b_{k}=0 \quad$ com $\quad b_{i}=b_{i 0}+b_{i 1} T+\ldots+b_{i s_{i}} T^{s_{i}}, b_{i j} \in I^{j}$. Substituindo essas expressões na equação satisfeita por $f T^{n}$ e tomando o coeficiente de $T^{n k}$, obtemos

$$
f^{k}+b_{1 n} f^{k-1}+b_{2(2 n)} f^{k-2}+\ldots+b_{k(k n)}=0, \quad b_{s(s n)} \in I^{s n}
$$

que é uma relação de dependência inteira de $f$ sobre $I^{n}$. Logo, $f \in \overline{I^{n}}$ e $J_{n} \subset \overline{I^{n}}$. Reciprocamente, se $f \in \overline{I^{n}}$, então existe $f^{k}+b_{1} f^{k-1}+\ldots+b_{k}=0$, com $b_{s} \in I^{n s}$. Daí,

$$
\left(f T^{n}\right)^{k}+b_{1} T^{n}\left(f T^{n}\right)^{k-1}+\ldots+b_{k} T^{n k}=0 \quad \text { com } \quad b_{s} T^{n s} \in I^{n s} T^{n s} .
$$

Assim, $f T^{n} \in \overline{\mathcal{P}(I)}$. Sendo $f T^{n}$ homogêneo de grau $n$ em $\mathcal{P}(I), f \in J_{n}$. Portanto, $\overline{I^{n}} \subset J_{n} \quad$ e $\overline{\mathcal{P}(I)}=\bigoplus_{n \in \mathbb{N}} \overline{I^{n}} T^{n}$.

Corolário 2.1.6. Dado um ideal I de R, temos:

(i). $(\bar{I})^{n} \subset \overline{I^{n}}$;

(ii) $I \overline{I^{n}} \subset \overline{I^{n+1}}$;

(iii) $\overline{\bar{I}}=\bar{I}$. 
Demonstração: (i) Dado $f \in(\bar{I})^{n}$, podemos supor que $f=g_{1} g_{2} \ldots g_{n}$ com $g_{j} \in$ $\bar{I}, j \in\{1, \ldots, n\}$. Então, $g_{j} T \in \overline{\mathcal{P}(I)}=\bigoplus_{d \in \mathbb{N}} \overline{I^{d}} T^{d}$. Sendo $g_{j} T$ um elemento homogêneo de grau 1 de $\mathcal{P}(I), g_{j} T \in \bar{I} T, \quad j \in\{1, \ldots, n\}$. Daí,

$$
f T^{n}=g_{1} T \ldots g_{n} T \in \bar{I} T \ldots \bar{I} T \subset \overline{I^{n}} T^{n} .
$$

Segue de $f T^{n}$ ser um elemento homogêneo que, $f \in \overline{I^{n}}$. Portanto, $(\bar{I})^{n} \subset \overline{I^{n}}$.

(ii) Se $f \in I \overline{I^{n}}$, podemos supor que $f=a g$ com $a \in I \subset \bar{I}$ e $g \in \overline{I^{n}}$. Então, $a T \in \bar{I} T, \quad g T^{n} \in \overline{I^{n}} T^{n}$. Segue da definição de álgebra graduada que

$$
f T^{n+1}=a T g T^{n} \in \overline{I^{n+1}} T^{n+1} .
$$

Assim, $f \in \overline{I^{n+1}}$ e $I \overline{I^{n}} \subset \overline{I^{n+1}}$.

(iii) Dado $f \in \overline{\bar{I}}$, temos que $f T \in \overline{\mathcal{P}(\bar{I})}$. Decorre do item (i) deste corolário que,

$$
\mathcal{P}(\bar{I})=\bigoplus_{n \in \mathbb{N}}(\bar{I})^{n} T^{n} \quad \subset \quad \bigoplus_{n \in \mathbb{N}} \overline{I^{n}} T^{n}=\overline{\mathcal{P}(I)} .
$$

Então, $\overline{\mathcal{P}(\bar{I})} \subset \overline{\overline{\mathcal{P}(I)}}=\overline{\mathcal{P}(I)}$. Assim, $f T \in \overline{\mathcal{P}(I)}, f \in \bar{I} \quad$ e $\overline{\bar{I}} \subset \bar{I}$. Como a inclusão contrária é trivial, $\overline{\bar{I}}=\bar{I}$.

Proposição 2.1.7. $f$ é inteiro sobre I se, e somente se, existe um R-módulo finitamente gerado $M$ tal que

(i) $f M \subset I M$

(ii) se $a M=0$, então existe $k \geq 0$ tal que $a f^{k}=0$.

Observação 2.1.8. Se $I$ é um ideal finitamente gerado e contém um elemento não divisor de zero, então a condição (ii) da proposição anterior é equivalente a: $M$ é um $R$ - módulo fiel, ou seja, $a M=0 \Leftrightarrow a=0$.

Demonstração: Se $f \in \bar{I}$, consideremos $f^{k}+a_{1} f^{k-1}+\ldots+a_{k}=0$ uma relação de dependência inteira de $f$ sobre $I$. Como $a_{j} \in I^{j}$, temos $a_{j}=\sum_{i} c_{1_{i}}^{j} \ldots c_{i_{j}}^{j} \operatorname{com} c_{i_{l}}^{j} \in I$ para todo $l \in\{1, \ldots, i\}$. Consideremos $S=\left\{c_{i_{l}}^{j}: 1 \leq l \leq j, 1 \leq j \leq k\right\}$ e $I_{0}=(S)$. $I_{0}$ é um ideal finitamente gerado em $R$, contido em $I$ e $v_{I_{0}}\left(a_{j}\right) \geq j$ para todo $j \in$ $\{1, \ldots, k\}$. 
Observamos que $f^{k} \in I_{0}\left(I_{0}+f R\right)^{k-1}$. De fato, segue de $\left(I_{0}+f R\right)^{k-1}=I_{0}^{k-1}+$ $I_{0}^{k-2} f R+\ldots+f^{k-1} R^{k-1}$ que,

$$
I_{0}\left(I_{0}+f R\right)^{k-1}=I_{0}^{k}+I_{0}^{k-1} f R+\ldots+I_{0} f^{k-1} R^{k-1} .
$$

Como $f^{k}=-a_{k}-a_{k-1} f-\ldots-a_{2} f^{k-2}-a_{1} f^{k-1}$ com $-a_{s} \in I_{0}^{s}, \quad f^{k} \in I_{0}\left(I_{0}+f R\right)^{k-1}$. Além disso,

$$
\begin{aligned}
\left(I_{0}+f R\right)^{k} & =I_{0}^{k}+I_{0}^{k-1} f R+\ldots+I_{0} f^{k-1} R+f^{k} R \\
& =I_{0}\left(I_{0}+f R\right)^{k-1}+f^{k} R \\
& \subset I_{0}\left(I_{0}+f R\right)^{k-1} .
\end{aligned}
$$

Consideremos $M=\left(I_{0}+f R\right)^{k-1}$. Então, $M$ é um ideal de $R$ finitamente gerado, logo, um $R$ - módulo. Mais ainda, $M$ satisfaz, as condições (i) e (ii) da proposição pois,

$$
f M \subset\left(I_{0}+f R\right) M=\left(I_{0}+f R\right)^{k} \subset I_{0}\left(I_{0}+f R\right)^{k-1} \subset I M
$$

e, se $0=a M=a\left(I_{0}+f R\right)^{k-1}$, decorre de $f^{k} \in I_{0}\left(I_{0}+f R\right)^{k-1}$ que $a f^{k} \in I_{0} a M=\{0\}$. Portanto, $a f^{k}=0$.

Reciprocamente, dado $\left\{m_{1}, \ldots, m_{s}\right\}$ um sistema de geradores de $M$, segue do item (i) da hipótese, que

$$
f m_{i}=\sum_{j=1}^{s} b_{i j} m_{j}, \quad \text { com } \quad b_{i j} \in I, \quad i, j \in\{1, \ldots, s\} .
$$

Observando que $f m_{i}=\sum_{j=1}^{s} f \delta_{i j} m_{j} \quad$ com $\quad \delta_{i j}=\left\{\begin{array}{cc}0, & i \neq j \\ 1, & i=j\end{array}\right.$, obtemos

$$
\begin{aligned}
0 & =f m_{i}-\sum_{j=1}^{s} b_{i j} m_{j}=\sum_{j=1}^{s} f \delta_{i j} m_{j}-\sum_{j=1}^{s} b_{i j} m_{j} \\
& =\sum_{j=1}^{s}\left(f \delta_{i j}-b_{i j}\right) m_{j} .
\end{aligned}
$$

Chamando $A=\left(a_{i j}\right), \quad$ com $a_{i j}=f \delta_{i j}-b_{i j}, \quad$ temos $A\left[\begin{array}{c}m_{1} \\ \vdots \\ m_{s}\end{array}\right]=\left[\begin{array}{c}0 \\ \vdots \\ 0\end{array}\right]$. Daí, 


$$
\left[\begin{array}{c}
0 \\
\vdots \\
0
\end{array}\right]=A^{t}\left[\begin{array}{c}
0 \\
\vdots \\
0
\end{array}\right]=A^{t} A\left[\begin{array}{c}
m_{1} \\
\vdots \\
m_{s}
\end{array}\right]=(\operatorname{det} A) I d\left[\begin{array}{c}
m_{1} \\
\vdots \\
m_{s}
\end{array}\right]=\left[\begin{array}{c}
\operatorname{det} A m_{1} \\
\vdots \\
\operatorname{det} A m_{s}
\end{array}\right] .
$$

Assim, $\operatorname{det} A m_{j}=0$ para $1 \leq j \leq s \quad$ e, conseqüentemente, $\operatorname{det} A M=0$. Segue do item (ii) da hipótese, que existe $k \geq 0$ tal que $\operatorname{det} A f^{k}=0$. Analisando a expressão $\operatorname{de} \operatorname{det} A$, obtemos uma relação de dependência inteira de $f$ sobre $I$. Portanto, $f \in \bar{I}$.

Corolário 2.1.9. Sejam $I, J$ ideais de R. Então, $\bar{I} \bar{J} \subset \overline{I J}$.

Demonstração: Como $\overline{I J}$ é ideal, é suficiente mostrar que dados $f \in \bar{I}, g \in$ $\bar{J}, \quad f g \in \overline{I J}$. Mostraremos que existe um $R$-módulo finitamente gerado satisfazendo as condições $(i)$ e (ii) da Proposição 2.1 .7 com relação ao elemento $f g$. Como $f \in \bar{I}, g \in \bar{J}$, existem $R$-módulos $M, N$ do tipo finito que satisfazem as condições (i), (ii) da referida proposição com respeito a $f, g$, respectivamente. Então, $S=M N$ é um $R$-módulo finitamente gerado. Além disso,

$$
f g S=f g M N=f M g N \subset I M . J N=I J S .
$$

Por outro lado, se $a S=0$ e $\left\{m_{1}, \ldots, m_{s}\right\}$ é um sistema de geradores de $M$, então $a m_{i} N=0 \quad \forall \quad i \in\{1, \ldots, s\}$. Daí, existe $r_{i} \geq 0, \quad$ tal que $a m_{i} g^{r_{i}}=0$.

Se $r=\max \left\{r_{1}, \ldots, r_{s}\right\}$, então $a g^{r} M=0$ e, existe $l \geq 0$ tal que $a g^{r} f^{l}=0$. Daí, $0=a(g f)^{\max \{k, l\}}$. Segue da Proposição 2.1 .7 que $f g \in \overline{I J}$. Portanto, $\bar{I} \bar{J} \subset \overline{I J}$.

Lema 2.1.10. Seja $R$ um anel noetheriano. Para todo ideal $I$ de $R$, as seguintes condições são equivalentes:

(i) $\bigoplus_{n \in \mathbb{N}} \overline{I^{n}} T^{n}\left[\right.$ respectivamente, $\left.\bigoplus_{n \in \mathbb{N}} \bar{I}^{n} T^{n}\right]$ é um $\mathcal{P}(I)$-módulo de tipo finito.

(ii) Existe um inteiro $N$ tal que se $n \geq N$, então $I \overline{I^{n}}=\overline{I^{n+1}}\left[I \bar{I}^{n}=\bar{I}^{n+1}\right]$.

Demonstração: Se $\overline{\mathcal{P}(I)}=\bigoplus_{n \in \mathbb{N}} \overline{I^{n}} T^{n}$ é um $\mathcal{P}(I)$-módulo de tipo finito, então consideremos $E_{1}, \ldots, E_{s}$ um sistema de geradores de $\overline{\mathcal{P}(I)}$. Sem perda de generalidade, 
podemos supor que cada $E_{j}$ é homogêneo. Denotemos $n_{i}=\operatorname{grau} E_{i}$, para $i \in\{1, \ldots, s\}$ e $N=\sup _{1 \leq i \leq s}\left\{n_{i}\right\}$. Dados $n \geq N$ e $f \in \overline{I^{n}}$, observamos que

$$
f T^{n+1}=\sum_{i=1}^{s} A_{i} E_{i} \quad \text { com } \quad A_{i} \in \mathcal{P}(I)=\bigoplus_{k \in \mathbb{N}} I^{k} T^{k}, \quad i \in\{1, \ldots, s\}
$$

e cada $A_{i}$ é homogêneo de grau $n+1-n_{i}$. De fato, segue de $E_{i}=e_{i} T^{n_{i}}$ com $e_{i} \in \overline{I^{n_{i}}} \mathrm{e}$ $A_{i}=a_{s_{1}} T^{s_{1}}+a_{s_{2}} T^{s_{2}}+\ldots+a_{s_{k}} T^{s_{k}}$, que $f T^{n+1}=\sum_{i=1}^{s} A_{i} E_{i}=\sum_{i=1}^{s} e_{i}\left(\sum_{j=1}^{k} a_{s_{j}} T^{n_{j}+s_{j}}\right)$. Como $f T^{n+1}$ é homogêneo de grau $n+1, a_{s_{j}}=0$ ou $n_{i}+s_{j}=n+1$. Ou seja, $A_{i}$ é homogêneo de grau $n+1-n_{i}$. Assim,

$$
\begin{aligned}
f \in \sum_{i=1}^{s} I^{n+1-n_{i}} \overline{I^{n_{i}}} & \subset I \sum_{i=1}^{s} I^{n-n_{i}} \overline{I^{n_{i}}} \\
& \subset I \sum_{i=1}^{s} \overline{I^{n-n_{i}+n_{i}}}=I \overline{I^{n}}
\end{aligned}
$$

e $\overline{I^{n+1}} \subset I \overline{I^{n}}$. Sendo a inclusão contrária trivial, $\overline{I^{n+1}}=I \overline{I^{n}}$. Analogamente, mostrase que se $\mathcal{P}(\bar{I})$ é um $\mathcal{P}(I)$-módulo do tipo finito, então $(\bar{I})^{n+1}=I(\bar{I})^{n}$.

Para mostrar a recíproca, observamos que existe $N \in \mathbb{N}$ tal que $\underset{s \in \mathbb{N}}{\bigoplus} \overline{I^{s}} T^{s}=$ $\bigoplus_{n \leq N} \overline{I^{n}} T^{n}+\overline{I^{N}} \mathcal{P}(I)$. De fato, como $R$ é noetheriano, podemos escolher $N \in \mathbb{N}$ satisfazendo a hipótese tal que $\overline{I^{s}}=\overline{I^{N}}$ para qualquer $s \geq N$. Daí,

$$
\begin{aligned}
\bigoplus_{n \leq N} \overline{I^{n}} T^{n}+\overline{I^{N}} \mathcal{P}(I) & =\bigoplus_{n \leq N} \overline{I^{n}} T^{n}+\bigoplus_{k \in \mathbb{N}} \overline{I^{N}} I^{k} T^{k} \\
& =\bigoplus_{n \leq N} \overline{I^{n}} T^{n}+\bigoplus_{k \in \mathbb{N}} \overline{I^{N+k}} T^{k} \\
& =\bigoplus_{n \leq N} \overline{I^{n}} T^{m}+\bigoplus_{k>N} \overline{I^{N+k}} T^{k} \\
& =\bigoplus_{n \leq N} \overline{I^{n}} T^{n}+\bigoplus_{k>N} \overline{I^{k}} T^{k} \\
& =\bigoplus_{s \leq N} \overline{I^{s}} T^{s} .
\end{aligned}
$$

Sendo $R$ noetheriano, $R[T]$ também o é, daí, todos os seus ideais são finitamente gerados. Em particular,

$$
\overline{I^{n}}=a_{1}^{n} \mathcal{P}(I) \oplus a_{2}^{n} \mathcal{P}(I) \oplus \ldots \oplus a_{m_{n}}{ }^{n} \mathcal{P}(I), \quad \forall \quad n \leq N .
$$

Assim, $\underset{s \in \mathbb{N}}{\oplus} \overline{I^{s}} T^{s}$ possui uma quantidade finita de geradores, isto é, $\overline{\mathcal{P}(I)}$ é um $\mathcal{P}(I)$ módulo finitamente gerado. 


\subsection{A Função de ordem $\bar{v}_{I}$}

Na seção anterior, expressamos nosso desejo de construir uma função de ordem $\mu$ tal que

$$
\mu(x) \geq 1 \quad \text { se, e somente se, } \quad x \in \bar{I} .
$$

Uma vez encontrada essa função de ordem, observamos que

$$
v_{I}(x) \geq 1 \Leftrightarrow x \in I \subset \bar{I} \Rightarrow \mu(x) \geq 1
$$

Daí, é razoável esperar que $\mu(x) \geq v_{I}(x)$. Se desejarmos que $\mu$, além de ser uma função de ordem, comporte-se como uma valoração, no sentido que $k \mu(x)=\mu\left(x^{k}\right) \geq v_{i}\left(x^{k}\right)$ para todo $x \in R, k \in \mathbb{N}$. Ou seja, $\mu(x) \geq \frac{v_{I}\left(x^{k}\right)}{k}$. Definimos então, $\mu: R \rightarrow \overline{\mathbb{R}}_{0}$ como $\mu(x):=\sup _{k \in \mathbb{N}}\left\{\frac{v_{I}\left(x^{k}\right)}{k}\right\}$.

A seguir, mostramos que $\mu$ é uma função de ordem e apresentamos algumas de suas propriedades. Na seção 2.3, mostramos que no caso em que $I$ é analílico $\mu$ satisfaz 2.3. Estando $\mu$ tão relacionada com o fecho integral do ideal $I$, é natural denotarmos

$$
\bar{v}_{I}(x)=\mu(x)=\sup _{k \in \mathbb{N}}\left\{\frac{v_{I}\left(x^{k}\right)}{k}\right\} .
$$

Dado um ideal $J$ de $R$, definimos $v_{I}\left(J^{k}\right):=\sup _{k \in \mathbb{N}}\left\{n \in \mathbb{N}:\left(J^{k}\right)^{n} \subset I\right\}$.

Lema 2.2.1. Sejam $R$ um anel e $I$ um ideal próprio de $R$. Dado um ideal $J$ de $R$, a seqüência $\left(u_{k}\right)_{k \in \mathbb{N}} \quad$ com $u_{k}=\frac{v_{I}\left(J^{k}\right)}{k}, k \in \mathbb{N}$, converge em $\overline{\mathbb{R}}_{0}$.

Demonstração: Sejam $\bar{u}, \underline{u}$ os limites superior e inferior, respectivamente, da seqüência $\left(u_{k}\right)_{k \in \mathbb{N}}$. Mostremos que $\bar{u}=\underline{u}$.

Se $\bar{u}=0$ ou $\underline{u}=\infty$, então $\underline{u}=\bar{u}$ pois $\underline{u} \leq \bar{u}$. Suponhamos então, que $\underline{u}$ seja finito e $\bar{u}>0$. Segue da definição que :

(i) dados $\epsilon>0, i \in \mathbb{N}$, existe $j \geq i$ tal que $u_{j}<\underline{u}+\epsilon$

(ii) dados $\epsilon>0, i \in \mathbb{N}$, existe $j \geq i$ tal que $u_{j}>\bar{u}-\epsilon \quad$ [caso em que $\bar{u}<\infty$ ] 
Fixemos $\epsilon>0$ e consideremos $i \ggg 0$ tal que $\frac{1}{i}<\frac{\epsilon}{\bar{u}-\epsilon}$. Segue de (ii) que, existe $j \geq i$ tal que $u_{j}>\bar{u}-\epsilon$. Por outro lado, decorre de $(i)$ que, existe $k \geq$ ij tal que $u_{k}<\underline{u}+\epsilon$. Usando o algoritmo da divisão, temos $k=l j+q$ com $q<j$. Assim,

$$
\begin{aligned}
\underline{u}+\epsilon & >u_{k}=u_{l j+q}=\frac{v_{I}\left(J^{l j+q}\right)}{l j+q} \geq \frac{v_{I}\left(J^{l j}\right)}{l j+q} \\
& \geq l \frac{v_{I}\left(J^{j}\right)}{l j+q}=\frac{v_{I}\left(J^{j}\right)}{j}\left(1-\frac{q}{k}\right)=u_{j}\left(1-\frac{q}{k}\right) \\
& >(\bar{u}-\epsilon)\left(1-\frac{q}{k}\right) \geq(\bar{u}-\epsilon)\left(1-\frac{1}{i}\right) \\
& >(\bar{u}-\epsilon)\left(1-\frac{\epsilon}{\bar{u}-\epsilon}\right) \\
& =(\bar{u}-\epsilon)\left(\frac{\bar{u}-\epsilon-\epsilon}{\bar{u}-\epsilon}\right)=\bar{u}-2 \epsilon .
\end{aligned}
$$

Como $\epsilon$ foi tomado arbitrariamente, concluímos que $\bar{u}=\underline{u}$. Quando $\bar{u}=\infty$, a prova é similar. Logo, a seqüência $\left(u_{k}\right)_{k \in \mathbb{N}}$ converge em $\overline{\mathbb{R}}_{0}$.

Observamos que a seqüência $\left(u_{k}\right)_{k \in \mathbb{N}}$ não é sempre crescente. Entretanto, fixado $i \in \mathbb{N}$, a subseqüência $\left(\tilde{u}_{n}\right)_{n \in \mathbb{N}}$ com $\tilde{u}_{n}=u_{i^{n}}$ o é. Com isso, podemos mostrar que

$$
\lim _{k \rightarrow \infty} u_{k}=\sup _{k \in \mathbb{N}}\left\{u_{k}\right\} .
$$

De fato, podemos supor que existe $i_{0}$ tal que $u_{i_{0}}=\sup _{k \in \mathbb{N}}\left\{u_{k}\right\}$ pois do contrário, existe uma subseqüência não trivial convergindo para $\sup _{k \in \mathbb{N}}\left\{u_{k}\right\}$. Como a seqüência $\left(u_{k}\right)_{k}$ é convergente, teríamos $\lim _{k \rightarrow \infty} u_{k}=\sup _{k \in \mathbb{N}}\left\{u_{k}\right\}$. Definindo então, a subseqüência $v_{n}=u_{i_{0}^{n}}$, temos uma subseqüência crescente. Daí,

$$
\sup _{k \in \mathbb{N}}\left\{u_{k}\right\}=u_{i_{0}}=v_{1} \leq v_{2} \leq v_{3} \leq \ldots ;
$$

Assim, estas desigualdades tornam-se igualdades e $\left(v_{m}\right)_{m}$ é constante e, converge para $\sup _{k \in \mathbb{N}}\left\{u_{k}\right\}$. Sendo $\left(u_{k}\right)_{k}$ convergente, converge para $\sup _{k \in \mathbb{N}}\left\{u_{k}\right\}$. Podemos então, apresentar a seguinte

Definição 2.2.2. Sejam $R$ um anel e I um ideal próprio de $R$. Dados $x$ um elemento e $J$ um ideal de $R$, definimos

$$
\bar{v}_{I}(x)=\lim _{k \rightarrow \infty} \frac{v_{I}\left(x^{k}\right)}{k} \quad \text { e } \quad \bar{v}_{I}(J)=\lim _{k \rightarrow \infty} \frac{v_{I}\left(J^{k}\right)}{k} .
$$


Proposição 2.2.3. Sejam $R$ um anel e I um ideal próprio de $R$. Seja $J$ um ideal de $R$. Se $\left\{x_{1}, \ldots, x_{m}\right\}$ é um sistema de geradores de $J$, então

$$
\bar{v}_{I}(J)=\inf _{1 \leq i \leq m}\left\{\bar{v}_{I}\left(x_{i}\right)\right\} .
$$

Demonstração: Para cada $k \in \mathbb{N}, x_{i}^{k} \in J^{k}$. Daí, $v_{I}\left(J^{k}\right) \leq v_{I}\left(x_{i}^{k}\right), \frac{v_{I}\left(J^{k}\right)}{k} \leq \frac{v_{I}\left(x_{i}^{k}\right)}{k}$. Logo, $\bar{v}_{I}(J) \leq \bar{v}_{I}\left(x_{i}\right)$, para cada $i \in\{1, \ldots, m\}$. Portanto, $\bar{v}_{I}(J) \leq \inf _{1 \leq i \leq m}\left\{\bar{v}_{I}\left(x_{i}\right)\right\}$.

Por outro lado, $J^{k}$ é gerado por $\left\{x_{1}^{\alpha_{1}} \ldots x_{m}^{\alpha_{m}}: \alpha_{1}+\ldots+\alpha_{m}=k\right\}$. Então,

$$
v_{I}\left(J^{k}\right)=\inf _{\sum \alpha_{i}=k}\left\{v_{I}\left(x_{1}^{\alpha_{1}} \ldots x_{m}^{\alpha_{m}}\right)\right\} .
$$

Sejam $a_{1}, \ldots, a_{m} \in \mathbb{N}$ tais que $v_{i}\left(J^{k}\right)=v_{I}\left(x_{1}^{a_{1}} \ldots x_{m}^{a_{m}}\right)$. Fixado $\epsilon>0$, escolhamos $k_{0} \in \mathbb{N}$ tal que

$$
k>k_{0} \Rightarrow \bar{v}_{I}\left(x_{i}\right)-\epsilon \leq \frac{v_{I}\left(x_{I}^{k}\right)}{k}, \quad \forall i \in\{1, \ldots, m\} .
$$

Seja $N=\sup _{1 \leq i \leq m, a \leq k_{0}}\left\{a \bar{v}_{I}\left(x_{i}\right)-v_{I}\left(x_{i}^{a}\right)\right\}$. Então,

$$
\begin{aligned}
v_{I}\left(x_{1}^{a_{1}} \ldots x_{m}^{a_{m}}\right) & \geq \sum_{i=1}^{m} v_{I}\left(x_{i}^{a_{i}}\right)=\sum_{a_{i}>k_{0}} v_{I}\left(x_{i}^{a_{i}}\right)+\sum_{a_{i} \leq k_{0}} v_{I}\left(x_{i}^{a_{i}}\right) \\
& \geq \sum_{a_{i}>k_{0}}\left(a_{i} \bar{v}_{I}\left(x_{i}\right)-\epsilon a_{i}\right)+\sum_{a_{i} \leq k_{0}}\left(a_{i} \bar{v}_{I}\left(x_{i}\right)-N\right) .
\end{aligned}
$$

Como $a_{1}+\ldots+a_{m}=k, \quad \sum_{a_{i}>k_{0}} a_{i} \leq k$. Daí, $-\sum_{a_{i}>k_{0}} \epsilon a_{i} \geq-k \epsilon$. Assim,

$$
\begin{aligned}
v_{I}\left(x_{1}^{a_{1}} \ldots x_{m}^{a_{m}}\right) & \geq \sum_{a_{i}>k_{0}} a_{i} \bar{v}_{I}\left(x_{i}\right)-k \epsilon+\sum_{a_{i} \leq k_{0}} a_{i} \bar{v}_{I}\left(x_{i}\right)-\sum_{a_{i} \leq k_{0}} N \\
& \geq \sum_{i=1}^{m} a_{i} \bar{v}_{I}\left(x_{i}\right)-k \epsilon-N m \\
& \geq k \inf _{1 \leq i \leq m}\left\{\bar{v}_{I}\left(x_{i}\right)\right\}-k \epsilon-N m .
\end{aligned}
$$

Portanto, $\frac{v_{I}\left(J^{k}\right)}{k}=\frac{v_{I}\left(x_{1}^{a_{1}} \ldots x_{m}^{a_{m}}\right)}{k} \geq \inf _{1 \leq i \leq m}\left\{\bar{v}_{I}\left(x_{i}\right)\right\}-\epsilon-\frac{N m}{k}$. Como $N$ depende apenas de $\epsilon$, fazendo $k \rightarrow \infty$, temos

$$
\bar{v}_{I}(J)=\lim _{k \rightarrow \infty} \frac{v_{I}\left(J^{k}\right)}{k} \geq \inf _{1 \leq i \leq m}\left\{\bar{v}_{I}\left(x_{i}\right)\right\}-\epsilon .
$$

Sendo $\epsilon$ arbitrário, $\bar{v}_{I}(J) \geq \inf _{1 \leq i \leq m}\left\{\bar{v}_{I}\left(x_{i}\right)\right\}$. Portanto, segue a igualdade. 
Corolário 2.2.4. $\bar{v}_{I}$ é uma função de ordem.

Demonstração: Dados $x, y \in R$, consideremos o ideal $J=(x, y)$. Segue do fato de $x+y \in J, \quad$ que $\bar{v}_{I}(x+y) \geq \bar{v}_{I}(J)=\inf \left\{\bar{v}_{I}(x), \bar{v}_{I}(y)\right\}$. Além disso, decorre de $v_{I}\left((x y)^{k}\right) \geq v_{I}\left(x^{k}\right)+v_{I}\left(y^{k}\right)$, que

$$
\frac{v_{I}\left((x y)^{k}\right)}{k} \geq \frac{v_{I}\left(x^{k}\right)}{k}+\frac{v_{I}\left(\left(y^{k}\right)\right.}{k} .
$$

Logo, $\bar{v}_{I}(x y) \geq \bar{v}_{I}(x)+\bar{v}_{I}(y)$.

Finalmente, $\bar{v}_{I}(0)=\lim _{k \rightarrow \infty} \frac{v_{I}(0)}{k}=\infty \quad$ e $\quad \bar{v}_{I}(1)=\lim _{k \rightarrow \infty} \frac{v_{I}(1)}{k}=0$. Portanto, $\bar{v}_{I}$ é uma função de ordem.

Observação 2.2.5. Se $x$ é um elemento nilpotente de $R$, então $\bar{v}_{I}(x)=\infty$.

Proposição 2.2.6. Sejam $R$ um anel e $I$ um ideal próprio de $R$. Dados $J$ um ideal de $R$ e $k \in \mathbb{N}$ temos:

(i) $\bar{v}_{I}\left(J^{k}\right)=k \bar{v}_{I}(J)$,

(ii) $\bar{v}_{I^{k}}(J)=\frac{1}{k} \bar{v}_{I}(J)$.

Demonstração: (i) $\frac{\bar{v}_{I}\left(J^{k}\right)}{k}=\frac{1}{k} \lim _{n \rightarrow \infty} \frac{v_{I}\left(J^{k n}\right)}{n}=\lim _{n \rightarrow \infty} \frac{v_{I}\left(J^{k n}\right)}{k n}=\bar{v}_{I}(J)$. Portanto, $\bar{v}_{I}\left(J^{k}\right)=k \bar{v}_{I}(J)$.

Para mostrar o item (ii), observamos que $k v_{I^{k}}\left(J^{n}\right) \leq v_{I}\left(J^{n}\right)$ para quaisquer $n, k \in$ $\mathbb{N}$. De fato, se $b=v_{I^{k}}\left(J^{n}\right)$, então $J^{n} \subset\left(I^{k}\right)^{b}=I^{k b}$. Daí,

$$
v_{I}\left(J^{n}\right) \geq k b=k v_{I^{k}}\left(J^{n}\right) .
$$

Assim, $\quad v_{I^{k}}\left(J^{n}\right) \leq \frac{1}{k} v_{I}\left(J^{n}\right), \quad \frac{v_{I^{k}}\left(J^{n}\right)}{n} \leq \frac{1}{k} \frac{v_{I}\left(J^{n}\right)}{n} \quad \mathrm{e}$,

$$
\bar{v}_{I^{k}}(J)=\lim _{n \rightarrow \infty} \frac{v_{I^{k}}\left(J^{n}\right)}{n} \leq \frac{1}{k} \lim _{n \rightarrow \infty} \frac{v_{I}\left(J^{n}\right)}{n}=\frac{1}{k} \bar{v}_{I}(J) .
$$

Logo, $\bar{v}_{I^{k}}(J) \leq \frac{1}{k} \bar{v}_{I}(J)$.

Chamemos $v_{n}=v_{I}\left(J^{n}\right)$. Dividindo $v_{n}$ por $k$, obtemos $v_{n}=q k+r$ com $0 \leq r<k$. Como $J^{n} \subset I^{v_{n}} \subset I^{q k}=\left(I^{k}\right)^{q}, \quad v_{I^{k}}\left(J^{n}\right) \geq q=\frac{v_{n}-r}{k}$. Assim,

$$
v_{I^{k}}\left(J^{n}\right) \geq \frac{v_{n}-r}{k} \geq \frac{v_{n}-k+1}{k} .
$$


Fazendo $n \rightarrow \infty$, obtemos:

$$
\bar{v}_{I^{k}}(J)=\lim _{n \rightarrow \infty} \frac{v_{I^{k}}\left(J^{n}\right)}{n} \geq \lim _{n \rightarrow \infty} \frac{v_{n}-k+1}{k n}=\lim _{n \rightarrow \infty} \frac{1}{k} \frac{v_{I}\left(J^{n}\right)}{n}=\frac{\bar{v}_{I}(J)}{k} .
$$

Portanto, $\bar{v}_{I^{k}}(J)=\frac{\bar{v}_{I}(J)}{k}$.

\subsection{Ideais em $\mathcal{O}_{X}$}

Nesta seção, apresentamos o resultado mais importante deste capítulo, o qual é devido a B. Teissier, M. Lejeune e H. Hironaka e apresenta condições equivalentes para um elemento $f$ pertencer ao fecho integral de um ideal analítico $I$. Como corolário, obtemos que $f \in \bar{I}$ se, e somente se, $v_{I}(f) \geq 1$. A proposição que apresentamos a seguir, dá-nos parte desta equivalência.

Proposição 2.3.1. Se $f \in \bar{I}$, então $\bar{v}_{I}(f) \geq 1$.

Demonstração: Consideremos $f^{k}+a_{1} f^{k-1}+\ldots+a_{k}=0, v_{I}\left(a_{i}\right) \geq 1, i \in\{1, \ldots, k\}$ uma relação de dependência inteira de $f$ sobre $I$. Sendo $\bar{v}_{I}$ uma função de ordem,

$$
\begin{aligned}
k \bar{v}_{I}(f)=\bar{v}_{I}\left(f^{k}\right) & \geq \inf _{i \in\{1, \ldots, k\}} \bar{v}_{I}\left(a_{i} f^{k-i}\right) \\
& \geq \inf _{i \in\{1, \ldots, k\}}\left\{\bar{v}_{I}\left(a_{i}\right)+\bar{v}_{I}\left(f^{k-i}\right)\right\} \\
& \geq \inf _{i \in\{1, \ldots, k\}}\left\{\bar{v}_{I}\left(a_{i}\right)+(k-i) \bar{v}_{I}(f)\right\} .
\end{aligned}
$$

Como $\bar{v}_{I}\left(a_{i}\right) \geq v_{I}\left(a_{i}\right) \geq i$

$$
\begin{aligned}
k \bar{v}_{I}(f) & \geq \inf _{i \in\{1, \ldots, k\}}\left\{\bar{v}_{I}\left(a_{i}\right)+(k-i) \bar{v}_{I}(f)\right\} \\
& \geq \inf _{i \in\{1, \ldots, k\}}\left\{i+(k-i) \bar{v}_{I}(f)\right\} .
\end{aligned}
$$

Se $i_{0}$ é o índice onde o ínfimo é atingido, então $k \bar{v}_{I}(f) \geq i_{0}+\left(k-i_{0}\right) \bar{v}_{I}(f)$. Assim, $\bar{v}_{I}(f) \geq 1$.

Corolário 2.3.2. Se $J$ é um ideal de tipo finito e $J \subset \bar{I}$, então $\bar{v}_{I}(J) \geq 1$.

Demonstração: Com efeito, se $J=\left(x_{1}, \ldots, x_{n}\right)$, então

$$
\bar{v}_{I}(J)=\inf _{1 \leq i \leq n}\left\{\bar{v}_{I}\left(x_{i}\right)\right\} \geq \inf _{1 \leq i \leq n}\{1\}=1 .
$$


Proposição 2.3.3. Sejam $R$ um anel noetheriano local. Se $I_{1}$ e $I_{2}$ são dois ideais primários para o ideal maximal de $R$ que têm o mesmo fecho integral, então eles têm a mesma multiplicidade.

Demonstração: Se $\overline{I_{1}}=\overline{I_{2}}$, então $I_{2} \subset \overline{I_{1}}$. Daí, $\bar{v}_{I_{1}}\left(I_{2}\right) \geq 1$. Dado $\epsilon>0$, existe $N \in \mathbb{N}$ tal que se $n \geq N$, então $v_{I_{1}}\left(I_{2}^{n}\right) \geq n(1-\epsilon)$. Seja $m=\min \{k \in \mathbb{Z}: k \geq$ $n(1-\epsilon), v_{I_{1}}\left(I_{2}^{n}\right) \geq k \quad$ e $\left.I_{2}^{n} \subset I_{1}^{k}\right\}$. Sejam $e\left(I_{j}\right)$ a multiplicidade de $I_{j}, j \in\{1,2\}$ e $d$ a dimensão de $A$. Segue da definição de multiplicidade de um ideal primário que

$$
e\left(I_{2}^{n}\right)=n^{d} e\left(I_{2}\right) \geq m^{d} e\left(I_{1}\right)=e\left(I_{1}^{m}\right) .
$$

Assim, $\frac{e\left(I_{2}\right)}{e\left(I_{1}\right)} \geq\left(\frac{m}{n}\right)^{d}$. Como $m \geq n(1-\epsilon), \frac{e\left(I_{2}\right)}{e\left(I_{1}\right)} \geq(1-\epsilon)^{d}$. Sendo $\epsilon>0$ arbitrário, $e\left(I_{2}\right) \geq e\left(I_{1}\right)$.

Repetindo esse argumento e usando o fato de $I_{1} \subset \overline{I_{2}}$, mostramos a desigualdade contrária.

Definição 2.3.4. Um anel $R$ é normal quando é integralmente fechado no anel das frações totais $\operatorname{Tot}(R)$.

Lema 2.3.5. Sejam $R$ um anel normal e $f$ um elemento não divisor de zero. Então, $f R$ é um ideal integralmente fechado em $R$.

Demonstração: Dado $g \in \overline{f R}$, consideremos $g^{k}+a_{1} f g^{k-1}+\ldots+a_{k} f^{k}=0$ uma relação de dependência inteira de $g$ sobre $f R$. Sendo $f$ não divisor de zero em $R, f$ é invertível em $T o t(R)$, como também $f^{k}$. Denotando por $\left(f^{k}\right)^{-1}$ o inverso de $f^{k}$ em $\operatorname{Tot}(R)$, temos

$$
0=\left(f^{k}\right)^{-1} 0=\left(f^{k}\right)^{-1}\left(g^{k}+a_{1} f g^{k-1}+\ldots+a_{k} f^{k}\right)=\left(\frac{g}{f}\right)^{k}+a_{1}\left(\frac{g}{f}\right)^{k-1}+\ldots+a_{k}
$$

que é uma relação de dependência inteira do elemento $\frac{g}{f}$ sobre o anel $R$. Como $R$ é normal, $\frac{g}{f}=a \in R$. Assim, $g=\frac{f g}{f} \in f R, \quad$ e, $\overline{f R}=f R$.

Este lema, embora seja simples, é fundamental na demonstração que apresentamos para o teorema seguinte. 
Até o momento, trabalhamos com ideais próprios em um anel comutativo com unidade. A partir de agora, nos restringimos ao estudo do fecho integral de ideais analíticos. Fixemos um espaço analítico complexo $X$ e $x_{0}$ um ponto de $X$.

Teorema 2.3.6. / Teorema de Caracterização / Seja I um ideal de $\mathcal{O}_{X, x_{0}}$. As seguintes condições são equivalentes:

(i) $h \in \bar{I}$;

(ii) (Critério avaliativo) Para cada curva analítica $\varphi:(\mathbb{C}, 0) \rightarrow\left(X, x_{0}\right)$, $h \circ \varphi \in\left(\varphi^{*}(I)\right) \mathcal{O}_{1} ;$

(iii) (Condição de crescimento) Para cada escolha de geradores $\left\{g_{i}\right\}$ de $I$, existem uma vizinhança $U$ de $x_{0}$ e uma constante $\mathcal{C}>0$ tal que,

$$
|h(x)| \leq \mathcal{C} \sup \left|g_{i}(x)\right| \quad \forall \quad x \in U
$$

(iv) Existe um $\mathcal{O}_{X, x_{0}}$-módulo fiel do tipo finito $L$ tal que h.L $\subset$ I.L.

A seguir, trabalhamos com o objetivo de demonstrar o Teorema 2.3.6. Para isso, vejamos alguns resultados que, uma vez provados, tornam simples a demonstração do teorema que aqui apresentamos.

Proposição 2.3.7. Sejam $X$ um espaço analítico reduzido e $x$ um ponto de $X$. Suponhamos que $X$ seja normal em uma vizinhança de $x$ e consideremos $f$ um germe de função meromorfa, não holomorfa, em uma vizinhança de $x$. Então, existe um germe de curva analítica complexa $h:(\mathbb{C}, 0) \rightarrow(X, x)$ tal que $f \circ h$ é um germe de função meromorfa não holomorfa na origem de $\mathbb{C}$.

Demonstração: Seja $\Pi: \tilde{X} \rightarrow X$ uma resolução de singularidades de $X$. Whitney, mostrou em [23] que $\overline{\mathcal{O}_{X}(U)}=\left\{\frac{g}{h}\right.$ meromorfa : $\frac{g}{h}$ é limitada $\}$. Sendo $X$ normal em uma vizinhança de $x$, existe uma vizinhança $U$ de $x$ tal que $\overline{\mathcal{O}_{X}(U)}=\mathcal{O}_{X}(U)$. Como $f$ é meromorfa não holomorfa, $f$ é não limitada em uma vizinhança de $x$. Daí, $f \circ \Pi$ é um germe de função meromorfa em uma vizinhança de $\Pi^{-1}(x)$ e existe $\tilde{x} \in \Pi^{-1}(x)$ tal que $f \circ \Pi$ não é holomorfa numa vizinhança de $\tilde{x}$. 
Então, podemos supor que $X$ é não singular em uma vizinhança de $x$. Sendo $X$ regular em $x$, o anel local $\mathcal{O}_{X, x}$, de $X$ em $x$, é regular, logo um domínio fatorial [ver [5], pag. 487]. Consideramos então, um representante do germe $f$ da forma $\frac{p}{q} \operatorname{com} p$ e $q$ holomorfas numa vizinhança de $x$ e sem fatores irredutíveis em comum, isto é, $p$ e $q$ primos entre si.

Observamos que o conjunto dos zeros de $q, q^{-1}(0)=\{y: q(y)=0\}$, contém uma hipersuperfície numa vizinhança de $x$ que não está contida no conjunto dos zeros de $p, p^{-1}(0)=\{y: q(y)=0\}$. De fato, se $Z(q)=q^{-1}(0) \subset p^{-1}(0)=Z(p)$, segue do Teorema do Zeros de Hilbert que $\sqrt{(q)}=I(Z(q)) \supset I(Z(p))=\sqrt{(p)}$. Mas, isso não ocorre pois $p$ e $q$ são primos entre si. Então, existe um germe de curva $\tilde{h}:(\mathbb{C}, 0) \rightarrow\left(\mathbb{C}^{n}, x\right)$ tal que $\operatorname{Im} \tilde{h} \subset q^{-1}(0)$ e $\operatorname{Im} \tilde{h} \not \subset p^{-1}(0)$ (ver [13]).

Identificando $\mathcal{O}_{\mathbb{C}, 0}$ com $\mathbb{C}\{t\}$ e denotando por $v$ a valoração natural em $\mathbb{C}\{t\}$, temos

$$
v(p \circ \tilde{h})=\alpha \quad \text { e } \quad v(q \circ \tilde{h})=\infty \quad \text { pois } q \circ \tilde{h} \equiv 0 .
$$

Queremos encontrar um germe de curva $h$ tal que $f \circ \tilde{h}=\frac{p \circ \tilde{h}}{q \circ \tilde{h}}$ seja meromorfa não holomorfa. Então, modifiquemos a curva $\tilde{h}=\left(\tilde{h_{1}}, \ldots, \tilde{h_{n}}\right)$ a fim de obter $h:(\mathbb{C}, 0) \rightarrow$ $\left(\mathbb{C}^{n}, x\right) \operatorname{com} v(p \circ h)=\alpha, \quad v(q \circ h)=\beta \quad$ e $\beta>\alpha$.

Para cada $N \in \mathbb{N}$, temos:

$$
\begin{aligned}
& p\left(\tilde{h}_{1}(t)+t^{N}, \tilde{h}_{2}(t), \ldots, \tilde{h}_{n}(t)\right)-p\left(\tilde{h}_{1}(t), \tilde{h}_{2}(t), \ldots, \tilde{h}_{n}(t)\right)=t^{N} \tilde{p}(t) \quad \text { com } \quad \tilde{p}(t) \in \mathbb{C}\{t\}, \\
& q\left(\tilde{h}_{1}(t)+t^{N}, \tilde{h}_{2}(t), \ldots, \tilde{h}_{n}(t)\right)-q\left(\tilde{h}_{1}(t), \tilde{h}_{2}(t), \ldots, \tilde{h}_{n}(t)\right)=t^{N} \tilde{q}(t) \quad \text { com } \quad \tilde{q}(t) \in \mathbb{C}\{t\}
\end{aligned}
$$

Em particular, se $N>\alpha$, então

$$
\begin{gathered}
v\left(p\left(\tilde{h}_{1}(t)+t^{N}, \tilde{h}_{2}(t), \ldots, \tilde{h}_{n}(t)\right)\right)=v\left(p\left(\tilde{h}_{1}(t), \tilde{h}_{2}(t), \ldots, \tilde{h}_{n}(t)\right)+t^{N} \tilde{p}(t)\right)=\alpha \quad e \\
v\left(q\left(\tilde{h}_{1}(t)+t^{N}, \tilde{h}_{2}(t), \ldots, \tilde{h}_{n}(t)\right)\right)=v\left(q\left(\tilde{h}_{1}(t), \tilde{h}_{2}(t), \ldots, \tilde{h}_{n}(t)\right)+t^{N} \tilde{q}(t)\right) \geq N>\alpha .
\end{gathered}
$$

Tomando $h:(\mathbb{C}, 0) \rightarrow(X, x), h=\left(h_{1}, \ldots, h_{n}\right)$ com

$$
h_{1}(t)=\tilde{h}_{1}(t)+t^{\alpha+1}, h_{i}(t)=\tilde{h}_{i}(t), i \geq 2,
$$

está demonstrada a proposição. 
No que segue, consideramos $X$ um espaço analítico complexo reduzido, $Y$ um subespaço analítico fechado e magro de $X$ e, $x$ um ponto de $Y$. Consideramos ainda, $\mathcal{I}$ o ideal coerente de $\mathcal{O}_{X}=\left\{\mathcal{O}_{X}(U): U=\tilde{U} \cap X, \tilde{U} \subset \mathbb{C}^{m}\right.$ aberto $\}$ que define $Y$. Denotamos por $I$ o germe de $\mathcal{I}$ em $x$.

Proposição 2.3.8. Seja $h \in \mathcal{O}_{X, x_{0}}$ tal que para cada germe de curva analítica $\varphi$ : $(\mathbb{C}, 0) \rightarrow\left(X, x_{0}\right)$, tem-se que $h \circ \varphi \in\left(\varphi^{*}(I)\right) \mathcal{O}_{1}$. Então para todo morfismo $\Pi: X^{\prime} \rightarrow$ $X$ satisfazendo:

(i) II própria e sobrejetora,

(ii) $X^{\prime}$ um espaço analítico normal,

(iii) $\Pi^{*}(\mathcal{I}) \mathcal{O}_{X^{\prime}}$ é um $\mathcal{O}_{X^{\prime}}$-módulo invertivel, existe um aberto $U$ de $X$ contendo $x$ tal que

$$
\Pi^{*}(h) \mathcal{O}_{\left.X^{\prime}\right|_{\Pi-1}(U)} \subset \Pi^{*}(\mathcal{I}) \mathcal{O}_{\left.X^{\prime}\right|_{\Pi^{-1}(U)}}
$$

Demonstração: Como II é própria, é suficiente mostrar que para todo $x^{\prime} \in \Pi^{-1}(x)$,

$$
\Pi^{*}(h) \mathcal{O}_{X^{\prime}, x^{\prime}} \in \Pi^{*}(I) \mathcal{O}_{X^{\prime}, x^{\prime}}
$$

Segue da condição (iii) que $\Pi^{*}(I) \mathcal{O}_{X^{\prime}, x^{\prime}}$ é invertível em $\mathcal{O}_{X^{\prime}, x^{\prime}}$. Daí, existe $g \in$ $\mathcal{O}_{X^{\prime}, x^{\prime}}$ não divisor de zero tal que $\Pi^{*}(I) \mathcal{O}_{X^{\prime}, x^{\prime}}=g \mathcal{O}_{X^{\prime}, x^{\prime}}$. Sendo $g$ não divisor de zero, podemos nos referir a $\frac{\Pi^{*}(h)}{g}$. Queremos mostrar que $\frac{\Pi^{*}(h)}{g} \in \mathcal{O}_{X^{\prime}, x^{\prime}}$.

Supondo o contrário, $\frac{\Pi^{*}(h)}{g}$ é meromorfa não holomorfa. Segue da Proposição 2.3.7 que existe $\gamma:(\mathbb{C}, 0) \rightarrow\left(X^{\prime}, x^{\prime}\right)$ tal que $\frac{\Pi^{*}(h)}{g} \circ \gamma$ é meromorfa não holomorfa. Isto é, $v\left(\gamma^{*}\left(\frac{\Pi^{*}(h)}{g}\right)\right)<0$, em que $v$ denota a valoração natural de $\mathbb{C}\{t\}$. Portanto, $v\left(\gamma^{*}\left(\Pi^{*}(h)\right)\right)<v\left(\gamma^{*}(g)\right)$.

Se $\varphi=\Pi \circ \gamma:(\mathbb{C}, 0) \rightarrow(X, x)$, então $\gamma^{*}(g)$ é um gerador de $\gamma^{*} \circ \Pi^{*}(I) \mathbb{C}\{t\}$. Daí, $\xi \in \gamma^{*}\left(\Pi^{*}(I)\right) \mathbb{C}\{t\}$ se, e somente se $v(\xi) \geq v\left(\gamma^{*}(g)\right)$. Por outro lado,

$$
v\left(\varphi^{*}(h)\right)=v\left((\Pi \circ \gamma)^{*}(h)\right)=v\left(\gamma^{*}\left(\Pi^{*}(h)\right)\right)<v\left(\gamma^{*}(g)\right) .
$$

Logo, $\varphi^{*}(h) \notin \gamma^{*}\left(\Pi^{*}(I)\right) \mathbb{C}\{t\}=\varphi^{*}(I) \mathbb{C}\{t\}$, contradizendo a hipótese feita sobre $h$. 
Lema 2.3.9. Sejam $(A, \eta)$ um anel noetheriano local e $J=\left(g_{1}, \ldots, g_{p}\right)$ um ideal principal distinto de (0). Então, existe $i \in\{1, \ldots, p\}$ tal que $J=\left(g_{i}\right)$.

Demonstração: Sendo $J$ principal, consideremos $g$ um gerador de $J$. Sejam $a_{1}, \ldots, a_{p}$, $b_{1}, \ldots, b_{p} \in A$ tais que

$$
g_{i}=a_{i} g \quad \text { e } \quad g=\sum_{i=1}^{p} b_{i} g_{i}
$$

Então, $g=\sum_{i=1}^{p} a_{i} b_{i} g$ e $g\left(1-\sum_{i=1}^{p} a_{i} b_{i}\right)=0$. Observamos que existe $i \in\{1, \ldots, p\}$ tal que $a_{i}$ é elemento invertível de $A$ pois do contrário, $\sum_{i=1}^{p} a_{i} b_{i} \in \eta, 1-\sum_{i=1}^{p} a_{i} b_{i}$ é invertível e $g=0$.

Assim, $g=a_{i}^{-1} g_{i}$ e $J=(g)=\left(g_{i}\right)$.

Lema 2.3.10. Sejam A um anel noetheriano de característica zero, $J$ um ideal contendo pelo menos um elemento não divisor de zero. Então, $J$ admite um sistema de geradores constituído de elementos não divisores de zero.

Demonstração: Sejam $h_{1} \in J$ um elemento não divisor de zero e $\left\{h_{1}, \ldots, h_{n}\right\}$ um sistema de geradores de $J$. Suponhamos que $h_{1}, \ldots, h_{k}$ sejam não divisores de zero. A estratégia que utilizamos nessa demonstração é modificar $h_{k+1}$ para obtermos um elemento não divisor de zero, de forma que, após um número finito de repetições deste processo, estejamos com um sistema de geradores constituído de elementos não divisores de zero.

Segue do fato de $A$ ser noetheriano que $A$ possui uma quantidade finita de ideais primos minimais e que um elemento é divisor de zero se, e somente se, pertence a um desses primos minimais. Para cada $s \in \mathbb{N}$, consideremos $g_{s}=h_{k+1}+s h_{k}$.

Suponhamos, por absurdo, que para todo $s \in \mathbb{N}, g_{s}$ é divisor de zero. Decorre do Princípio das Gavetas que existem $s_{1}, s_{2} \in \mathbb{N}$ e um primo minimal $P$ tais que $g_{s_{1}}, g_{s_{2}} \in P$. Então,

$$
g_{s_{1}}-g_{s_{2}}=\left(s_{1}-s_{2}\right) h_{k} \in P .
$$

Assim, $\left(s_{1}-s_{2}\right) h_{k}$ é divisor de zero, isto é, existe $a \neq 0$ tal que $0=a\left(s_{1}-s_{2}\right) h_{k}=$ $\left(s_{1}-s_{2}\right) a h_{k}$. Como $A$ tem característica zero, $a h_{k}=0$. Mas, isto contradiz o fato de 
$h_{k}$ ser não divisor de zero. Portanto, existe $s_{0} \in \mathbb{N}$ tal que $g_{s_{0}}$ é não divisor de zero. Substituindo $h_{k+1}$ por $g_{s_{0}}$ e, repetindo esse procedimento um número finito de vezes, mostramos o lema.

Proposição 2.3.11. Sejam $V$ uma vizinhança de $x_{0}$ para a qual $\mathcal{J}$ e $\mathcal{I}$ são gerados por seções globais. São equivalentes as seguintes afirmações:

(i) Para todo sistema de geradores $\left\{g_{1}, \ldots, g_{m}\right\}$ de $\Gamma(V, \mathcal{I})$ e todo elemento $f$ de $\Gamma(V, \mathcal{J})$, podemos encontrar uma vizinhança $V^{\prime}$ de $x_{0}$ e uma constante $\mathcal{C}$ tais que

$$
|f(y)| \leq \mathcal{C} \sup _{i=1, \ldots, m}\left|g_{i}(y)\right| \quad \forall \quad y \in V^{\prime} .
$$

(ii) Se o morfismo $\Pi: X^{\prime} \rightarrow X$ satisfaz:

(a) ПI é própria e sobrejetora,

(b) $X^{\prime}$ é um espaço analítico normal,

(c) $\Pi^{*}(\mathcal{I}) \mathcal{O}_{X^{\prime}}$ é um $\mathcal{O}_{X^{\prime}}$-módulo invertível,

então existe um aberto $U$ de $X$ contendo $x$ tal que

$$
\Pi^{*}(h) \mathcal{O}_{\left.X^{\prime}\right|_{\Pi^{-1}(U)}} \subset \Pi^{*}(\mathcal{I}) \mathcal{O}_{\left.X^{\prime}\right|_{\Pi^{-1}(U)}}
$$

Demonstração: Pelo Lema 2.3.10 podemos considerar $\left\{g_{1}, \ldots, g_{m}\right\}$ um sistema de geradores de $I$ formado de elementos não divisores de zero. Como II é própria, podemos recobrir $\Pi^{-1}(x)$ por um número finito de abertos $V_{\alpha}$ relativamente compactos sobre os quais um dos $g_{i}$ gera $\Pi^{*}(\mathcal{I}) \mathcal{O}_{\left.X^{\prime}\right|_{\Pi^{-1}\left(V_{\alpha}\right)}}$ [cf Lema 2.3.9]. Como $\Pi$ é sobrejetora, a condição $(i)$ é satisfeita se, e somente se, sobre cada $V_{\alpha}, \frac{|h \circ \Pi|}{\sup \left|g_{i} \circ \Pi\right|}$ é limitada. Ora, se $g_{1}$ é o gerador de $\Pi^{*}(\mathcal{I}) \mathcal{O}_{\left.X^{\prime}\right|_{\Pi^{-1}(U)}}$ sobre $V_{\alpha}$, então este último quociente é limitado se, e somente se, $\frac{|h \circ \Pi|}{\left|g_{1} \circ \Pi\right|}$ é limitado. Mas, em todo ponto $y \in V_{\alpha}$, o germe de $g_{1}$ é não divisor de zero em $\mathcal{O}_{X^{\prime}, y}$, daí, $\frac{f \circ \Pi}{g_{1} \circ \Pi}$ induz um elemento de $\operatorname{Tot}\left(\mathcal{O}_{X^{\prime}, y}\right)$. Como $X^{\prime}$ é um espaço normal, esta última frase equivale a $\frac{h \circ \Pi}{g_{1} \circ \Pi}$ pertencer a $\mathcal{O}_{X^{\prime}, y}$, para cada $y \in V_{\alpha}$, isto é, $\Pi^{*}(h) \mathcal{O}_{\left.X^{\prime}\right|_{\Pi^{-1}(U)}} \subset \Pi^{*}(\mathcal{I}) \mathcal{O}_{\left.X^{\prime}\right|_{\Pi^{-1}(U)}}$. 
Proposição 2.3.12. Suponhamos que para todo morfismo $\Pi: X^{\prime} \rightarrow X$ satisfazendo:

(i) П própria e sobrejetora,

(ii) $X^{\prime}$ um espaço analítico normal,

(iii) $\Pi^{*}(\mathcal{I}) \mathcal{O}_{X^{\prime}}$ é um $\mathcal{O}_{X^{\prime}}$-módulo invertível,

exista um aberto $U$ de $X$ contendo $x$ tal que

$$
\left.\left.\Pi^{*}(h) \mathcal{O}_{X^{\prime}}\right|_{\Pi^{-1}(U)} \subset \Pi^{*}(\mathcal{I}) \mathcal{O}_{X^{\prime}}\right|_{\Pi^{-1}(U)}
$$

Então, existe um $\mathcal{O}_{X}$-ideal coerente $\mathcal{K}$ com suporte raro em $X$ tal que se $\psi: \tilde{X} \rightarrow X$ é a explosão de $\mathcal{K}$ então,

$$
\psi^{*}(h) \in \psi^{*}(I) \mathcal{O}_{\tilde{X}, \tilde{x}} \quad \forall \quad \tilde{x} \in \psi^{-1}\left(x_{0}\right)
$$

Demonstração: Dado um espaço analítico reduzido $\mathcal{X}$, existe um ideal coerente sobre este espaço cuja explosão é a normalização de $\mathcal{X}$.

Seja $\Pi: \overline{X^{\prime}} \rightarrow X$ a explosão normalizada de $Y$. Consideremos $\mathcal{K} \circ \mathcal{O}_{X \text {-ideal }}$ coerente que podemos conseguir a partir da explosão de $Y$ e, cuja explosão é a normalização de $X$. Sendo $Y$ magro e $\Pi$ satisfazendo as condições $(i),(i i),(i i i)$, o resultado segue da hipótese.

Proposição 2.3.13. Seja $h \in \mathcal{O}_{X, x_{0}}$. Suponhamos que exista um $\mathcal{O}_{X}$-ideal coerente $\mathcal{K}$ com suporte raro em $X$ tal que se $\Pi: \tilde{X} \rightarrow X$ é a explosão de $\mathcal{K}$ então

$$
\Pi^{*}(h) \in \Pi^{*}(I) \mathcal{O}_{\tilde{X}, \tilde{x}}, \quad \forall \quad \tilde{x} \in \Pi^{-1}\left(x_{0}\right) .
$$

Nestas condições, temos que $h \in \bar{I}$.

Demonstração: Como $\mathcal{K}$ tem suporte raro, podemos utilizar o Lema 2.3 .10 e a coerência de $\mathcal{K}$ para supor que, para abertos $U$ suficientemente pequenos, $\mathcal{K}_{\left.\right|_{U}}$ seja gerado por um número finito de seções globais, $g_{1}, \ldots, g_{m}$ não divisoras de zero. Também, podemos supor que $U$ é suficientemente pequeno tal que $\mathcal{I}_{\mid U}$ seja gerado 
por seções globais. Então, $\Pi^{-1}(U)$ é recoberto por um número finito de abertos tais que:

$$
\mathcal{O}_{\left.\tilde{X}\right|_{V_{i}}} \simeq \mathcal{O}_{\left.X\right|_{U}}\left[\frac{g_{1}}{g_{i}}, \ldots, \frac{\widehat{g}_{i}}{g_{i}}, \ldots, \frac{g_{m}}{g_{i}}\right] .
$$

sobre cada aberto $V_{i}, h$ se exprime como um polinômio em função de $\frac{g_{k}}{g_{i}}, k \neq i$. Seja $K$ o germe de $\mathcal{K}$ em $x$ e, seja $n_{i} \geq 1$ um inteiro tal que

$$
h g_{i}^{n_{i}} \in I K^{n_{i}} .
$$

Tomemos $n=\sum_{i=1}^{m} n_{i} \quad$ e $\quad h g_{1}^{\alpha_{1}} \cdots g_{m}^{\alpha_{m}}$. Se $\alpha_{1}+\cdots+\alpha_{m} \geq n$, certamente existe $i_{0}$ tal que $\alpha_{i_{0}} \geq n_{i_{0}}$. Então, podemos escrever

$$
h g_{1}^{\alpha_{1}} \cdots g_{m}^{\alpha_{m}}=h g_{i_{0}}^{n_{i_{0}}} g_{i_{0}}^{\alpha_{i_{0}}-n_{i_{0}}} \prod_{j \neq i_{0}} g_{j}^{\alpha_{j}} \in I K^{n_{i_{0}}} K^{n-n_{i_{0}}}=K^{n}
$$

Isso mostra que $h K^{n} \subset I K^{n}$. Como $K$ contém um elemento não divisor de zero de $\mathcal{O}_{X, x}, K^{n}$ é um $\mathcal{O}_{X, x}$-módulo fiel de tipo finito. Daí, segue da Proposição 2.1.7 que $h \in \bar{I}$.

Observação 2.3.14. Para cada ideal próprio $J \neq(0)$ de $\mathbb{C}\{t\}$, existe um inteiro $m \geq 1$ tal que $J=t^{m} \mathbb{C}\{t\}$.

Demonstração do teorema 2.3.6: $(i) \Rightarrow(i i) \operatorname{Sejam} \varphi:(\mathbb{C}, 0) \rightarrow\left(X, x_{0}\right)$ uma curva analítica e denotemos por $v$ a valoração natural de $\mathbb{C}\{t\}$. Segue da observação feita acima que existe $m \geq 1$ tal que $\varphi^{*}(I) \mathbb{C}\{t\}=t^{m} \mathbb{C}\{t\}$. Então, para mostrar que $h \circ \varphi \in \varphi^{*}(I) \mathbb{C}\{t\}$, devemos mostrar que $v(h \circ \varphi) \geq m$. Mas, segue da Proposição 2.3.1 que

$$
\bar{v}_{\varphi^{*}(I) \mathbb{C}\{t\}}(h \circ \varphi) \geq \bar{v}_{I}(h) \geq 1
$$

pois $h \in \bar{I}$ e $\varphi^{*}$ é homomorfismo. Daí,

$$
1 \leq \bar{v}_{\varphi^{m}(I) \mathbb{C}\{t\}}(h \circ \varphi)=\bar{v}_{t^{m} \mathbb{C}\{t\}}(h \circ \varphi)=\frac{1}{m} \bar{v}_{t \mathbb{C}\{t\}}(h \circ \varphi)=\frac{1}{m} v(h \circ \varphi) .
$$

Portanto, $v(h \circ \varphi) \geq m$ e $h \circ \varphi \in \varphi^{*}(I) \mathbb{C}\{t\}$.

$($ ii $) \Rightarrow($ iii $)$ Decorre das proposições 2.3 .8 e 2.3.11.

(iii) $\Rightarrow$ (i) Decorre das proposições 2.3.11, 2.3.12 e 2.3.13. 
$(i) \Leftrightarrow(i v)$ Esta equivalência é exatamente o que diz a Proposição 2.1.7.

Observando a prova de $(i) \Rightarrow(i i)$, vemos que foi mostrado que se $\bar{v}_{I}(f) \geq 1$, então vale o critério avaliativo. Este, por sua vez, implica que $f \in \bar{I}$. Assim, segue da Proposição 2.3 .1 o seguinte

Corolário 2.3.15. $f \in \bar{I}$ se, e somente se, $\bar{v}_{I}(f) \geq 1$. 


\section{Capítulo 3}

\section{Filtração de Newton e não-degeneração}

\subsection{A filtração de Newton}

Para cada $g(x)=\sum a_{k} x^{k}$, o suporte de $g$ é definido como

$$
\operatorname{supp}(g)=\left\{k \in \mathbb{Z}^{n}: a_{k} \neq 0\right\} .
$$

Definição 3.1.1. Seja I um ideal em $\mathcal{O}_{n}$. Definimos $\operatorname{supp}(I)=\bigcup_{\mathbf{g} \in \mathbf{I}} \operatorname{supp}(g) \quad e$, $E(I)=\bigcup_{k \in \operatorname{supp}(I)}\left\{k+v: v \in \mathbb{R}_{+}^{n}\right\}$

Definição 3.1.2. O poliedro de Newton do ideal $I$, que denotamos por $\Gamma_{+}(I)$, é o fecho convexo em $\mathbb{R}_{+}^{n}$ do conjunto $E(I)$. Denotamos por $\Gamma(I)$ a união das faces compactas de $\Gamma_{+}(I)$.

A seguir, consideramos $I=\left(g_{1}, \ldots, g_{k}\right)$ um ideal de codimensão finita em $\mathcal{O}_{n}$, isto é, $\operatorname{dim}_{\mathbb{C}} \frac{\mathcal{O}_{n}}{I}<\infty$.

Dado um vetor $v \in \mathbb{R}_{+}^{n} \backslash\{0\}$, definimos $l(v)=\min \left\{\langle k, v\rangle: k \in \Gamma_{+}(I)\right\}$.

Uma definição formal para face de um poliedro é a seguinte: um subconjunto $\Delta$ de $\Gamma_{+}(I)$ é uma face quando existe $v \in \mathbb{R}_{+}^{n} \backslash\{0\}$ tal que $\Delta=\left\{k \in \Gamma_{+}(I):\langle k, v\rangle=l(v)\right\}$. Nesse caso, $v$ define a face $\Delta$. Quando $v$ define $\Delta$ e tem comprimento mínimo no conjunto $\mathbb{R}_{+}^{n} \backslash\{0\} \cap \mathbb{Z}_{+}^{n}$, v é chamado um vetor primitivo para $\Delta$. 
Dado um subconjunto finito $\Delta \subset \Gamma_{+}(I)$, para qualquer germe $g(x)=\sum a_{k} x^{k}$, definimos $g_{\Delta}=\sum_{k \in \Delta} a_{k} x^{k}$. Se $\Delta$ é uma face de $\Gamma_{+}(I), C(\Delta)$ denota o cone dos semiraios que partem da origem e passam por $\Delta$.

Sendo $I$ de codimensão finita e $\Gamma_{+}(I)$ um poliedro convexo em $\mathbb{R}_{+}^{n}$, a coleção de todos os $C(\Delta)$ nos fornece uma decomposição poliedral para $\mathbb{R}_{+}^{n}$.

No que segue, construímos uma filtração em $\mathcal{O}_{n}$ a partir do poliedro de Newton $\Gamma_{+}(I)$, a qual chamaremos de filtração de Newton.

Para motivar a construção de uma filtração de Newton em $\mathcal{O}_{n}$, lembramos o que ocorre na filtração homogênea.

Dado $f(x)=\sum a_{k} x^{k} \in \mathcal{O}_{n}$, escrevemos $f=f_{d}+f_{d+1}+\ldots$ em que $f_{i}$ é uma forma de grau $i$, isto é, um polinômio homogêneo de grau $i$. O grau de homogeneidade é medido pela valoração $v_{h}: \mathcal{O}_{n} \rightarrow \overline{\mathbb{R}}, \quad v_{h}(f)=\min \{\varphi(k): k \in \operatorname{supp}(f)\}$ em que $\varphi: \mathbb{R}_{+}^{n} \rightarrow \overline{\mathbb{R}}, \quad \varphi(k)=\varphi\left(k_{1}, \ldots, k_{n}\right)=k_{1}+\ldots+k_{n}$.

Analisemos o que ocorre quando $n=2$. Para cada $d \in \mathbb{R}, \varphi^{-1}(d)$ é a interseção da reta $x+y=d$ com $\mathbb{R}_{+}^{2}$.

$\mathrm{O}$ conjunto $\varphi^{-1}(d)$, que denotamos por $\Delta_{1}$ é a face de nível $d$ da filtração homogênea. Denotamos por $\Gamma$ o envoltório convexo em $\mathbb{R}_{+}^{2}$ do conjunto $\Delta_{1}+\mathbb{R}_{+}^{2}$.

Neste polígono, $\Delta_{1}$ é a única face compacta de dimensão 1 . Observamos que a função $\varphi$ satisfaz as seguintes propriedades:

(i) $\varphi$ é linear em $C\left(\Delta_{1}\right)$

(ii) $\varphi$ assume valores inteiros positivos em $\mathbb{Z}_{+}^{2} \cap \mathbb{R}_{+}^{2}$

(iii) $\varphi$ é constante na face compacta $\Delta_{1}$.

A fim de generalizar essa idéia, ao fixarmos um poliedro $\Gamma_{+}(I)$, obtemos uma aplicação

$$
\Phi: \mathbb{R}_{+}^{n} \rightarrow \overline{\mathbb{R}}
$$

satisfazendo as seguintes propriedades:

(i) para cada face $\Delta$ de $\Gamma_{+}(I), \Phi$ é linear em $C(\Delta)$, 
(ii) $\Phi$ assume valores inteiros positivos em $\mathbb{R}_{+}^{n} \cap \mathbb{Z}_{+}^{n}$,

(iii) $\exists p \in \mathbb{N}$ tal que $\Phi_{\mid \Gamma(I)}=p$.

Definimos $f i l: \mathcal{O}_{n} \rightarrow \overline{\mathbb{R}} \operatorname{com} f i l(f)=\inf \{\Phi(k): k \in \operatorname{supp}(f)\}$. Como supp $(0)=$ $\varnothing$, fil não está definida no germe nulo, convencionamos então $f i l(0)=\infty$.

Observamos que nestas condições, $\Phi\left(k_{1}+k_{2}\right) \geq \Phi\left(k_{1}\right)+\Phi\left(k_{2}\right)$ para quaisquer $k_{1}, k_{2} \in \mathbb{R}_{+}^{n}$. De fato, escrevemos $k_{1}=\lambda_{1} s_{1}, k_{2}=\lambda_{2} s_{2}$ com $\lambda_{1}, \lambda_{2} \in \mathbb{R}, s_{1}, s_{2}$ pertencendo a faces compactas de $\Gamma_{+}(I)$. Daí, $\Phi\left(k_{1}+k_{2}\right)=\Phi\left(\lambda_{1} s_{1}+\lambda_{2} s_{2}\right)=\left(\lambda_{1}+\lambda_{2}\right) \Phi\left(\frac{1}{\lambda_{1}+\lambda_{2}}\left(\lambda_{1} s_{1}+\lambda_{2} s_{2}\right)\right)=\left(\lambda_{1}+\lambda_{2}\right) \Phi(\beta s)$ $\operatorname{com} \beta \geq 1$ e $s$ pertencendo a uma face compacdta de $\Gamma_{+}(I)$, já que $\frac{1}{\lambda_{1}+\lambda_{2}}\left(\lambda_{1} s_{1}+\lambda_{2} s_{2}\right) \in$ $\Gamma_{+}(I)$. Assim,

$$
\Phi\left(k_{1}+k_{2}\right)=\left(\lambda_{1}+\lambda_{2}\right) \beta \Phi(s) \geq\left(\lambda_{1}+\lambda_{2}\right) p=\Phi\left(k_{1}\right)+\Phi\left(k_{2}\right) .
$$

Com isso, vale o seguinte

Lema 3.1.3. fil é uma função de ordem em $\mathcal{O}_{n}$.

Demonstração: Com efeito, dados $g, h \in \mathcal{O}_{n}$, lembrando que

$$
\operatorname{supp}(g+h) \subset \operatorname{supp}(g) \cup \operatorname{supp}(h),
$$

temos:

$$
\begin{aligned}
\operatorname{fil}(g+h) & =\inf \{\Phi(k): k \in \operatorname{supp}(g+h)\} \\
& \geq \inf \{\Phi(k): k \in \operatorname{supp}(g) \cup \operatorname{supp}(h)\} \\
& =\inf \{\inf \{\Phi(k): k \in \operatorname{supp}(g)\}, \inf \{\Phi(k): k \in \operatorname{supp}(h)\}\} \\
& =\inf \{f i l(g), f i l(h)\} .
\end{aligned}
$$

Além disso, como $\operatorname{supp}(g h) \subset \operatorname{supp}(g)+\operatorname{supp}(h)$,

$f i l(g h)=\inf \{\Phi(k): k \in \operatorname{supp}(g h)\}$

$$
\begin{aligned}
& \geq \inf \left\{\Phi\left(k_{1}\right)+\Phi\left(k_{2}\right): k_{1} \in \operatorname{supp}(g), k_{2} \in \operatorname{supp}(h)\right\} \\
& =\inf \left\{\Phi\left(k_{1}\right): k_{1} \in \operatorname{supp}(g)\right\}+\inf \left\{\Phi\left(k_{2}\right): k_{2} \in \operatorname{supp}(h)\right\} \\
& =\operatorname{fil}(g)+f i l(h) .
\end{aligned}
$$


Sendo $f i l(1)=\inf \{\Phi(k): k \in \operatorname{supp}(1)=\{0\}\}=\Phi(0)=0$ e fil $(0)=\infty$, concluímos que $f i l$ é uma função de ordem em $\mathcal{O}_{n}$.

Observação 3.1.4. Para cada $q \in \mathbb{N}$, consideremos o conjunto

$$
\mathcal{A}_{q}=\left\{g \in \mathcal{O}_{n}: \Phi(k) \geq q, \quad \forall \quad k \in \operatorname{supp}(g)\right\}=\left\{g \in \mathcal{O}_{n}: f i l(g) \geq q\right\}
$$

Sendo fil uma função de ordem, a seqüência decrescente

$$
\mathcal{O}_{n}=\mathcal{A}_{0} \supset \mathcal{A}_{1} \supset \ldots
$$

é uma filtração de $\mathcal{O}_{n}$ que denominamos a filtração de Newton de $\mathcal{O}_{n}$ associada a $\Gamma_{+}(I)$.

O exemplo a seguir ilustra a importância de considerarmos uma filtração de Newton ao invés da filtração homogênea.

Exemplo 3.1.5. Seja $f(x, y)=y^{2}+x^{3}+x^{7}$. A parte principal de $f$ com relação a filtração homogênea é $g(x, y)=y^{2}$, que não possui singularidade isolada na origem. Porém, se atribuirmos os pesos $w t(x)=2$ e $w t(y)=3$, a parte principal de $f$ será $y^{2}+x^{3}$ que possui singularidade isolada na origem. Para fins deste tipo, podemos utilizar o poliedro de Newton.

Em geral, encontramos uma filtração de Newton tal que a parte principal de $f$ em relação a esta, possua singularidade isolada na origem. No caso em que $f(x, y)=$ $y^{4}-2 x^{3} y^{2}+x^{12}$, isso não ocorre; ou seja, a parte principal de $f$ em relação a qualquer filtração não possui singularidade isolada na origem. Observamos que o conjunto dos germes cuja parte principal possui singularidade isolada com relação a algum poliedro de Newton é um conjunto genérico.

No exemplo abaixo mostramos como encontrar uma função : $\mathbb{R}_{+}^{n} \rightarrow \overline{\mathbb{R}}$ com as propriedades que impusemos para $\Phi$.

Exemplo 3.1.6. Considere o ideal $I=\left(x^{4}+x y^{3}, y^{5}+2 x^{6} y\right)$ de $\mathcal{O}_{2}$. O poliedro de Newton de $I$ é o conjunto em $\mathbb{R}_{+}^{2}$ cujos vértices são $(0,5),(1,3),(4,0)$.

Chamemos $\Delta_{1}$ e $\Delta_{2}$ as faces com vértices em $\{(0,5),(1,3)\},\{(1,3),(4,0)\}$, respectivamente. Essas são as faces compactas de dimensão 1 de $\Gamma_{+}(I)$. 
Temos que $v_{1}=(2,1)$ e $v_{2}=(1,1)$ são vetores primitivos com respeito a $\Delta_{1}, \Delta_{2}$, respectivamente. Além disso, $l\left(v_{1}\right)=5$ e $l\left(v_{2}\right)=4$.

Consideramos $M=$ m.m.c. $\left\{l\left(v_{1}\right), l\left(v_{2}\right)\right\}=20$. Definimos $\varphi_{i}: \mathbb{R}_{+}^{2} \rightarrow \mathbb{R}$ como

$$
\varphi_{i}(k)=\frac{M}{l\left(v_{i}\right)}<k, v_{i}>=\frac{20}{l\left(v_{i}\right)}<k, v_{i}>, \quad i=1,2 .
$$

Note que cada $\varphi_{i}$ é linear. Definimos também, $\varphi: \mathbb{R}_{+}^{2} \rightarrow \mathbb{R}$ por

$$
\varphi(k)=\min \left\{\frac{M}{l\left(v_{i}\right)}<k, v_{i}>: i=1,2\right\} .
$$

Observamos que $\varphi(k)=\varphi_{i}(k) \quad \forall k \in C\left(\Delta_{i}\right)$ pois,

$$
\varphi(k)=\varphi_{i}(\lambda s)=\lambda \frac{M}{l\left(v_{i}\right)}<s, v_{i}>
$$

para algum $s \in \Delta_{i} ;$ daí, $\varphi_{j}(k)=\lambda \frac{M}{l\left(v_{j}\right)}\left\langle s, v_{j}\right\rangle \geq \lambda M=\varphi_{i}(k)$. Logo, $\varphi$ é linear em cada cone $C\left(\Delta_{i}\right)$.

Além disso, $\varphi\left(\mathbb{Z}_{+}^{2}\right) \subset \mathbb{Z}_{+} \mathrm{e}, \varphi(k)=M$ para qualquer ponto $k$ pertencente a uma face compacta de $\Gamma_{+}(I)$. Portanto, $\varphi$ é uma função que vai gerar a filtração de Newton associada a $\Gamma_{+}(I)$ da maneira que descrevemos anteriormente.

Para cada $\Delta \in \Gamma(I)$, definimos o anel $\mathbb{C}[[\Delta]]$, consistindo dás séries de potências com monômios não nulos $x^{s}=x_{1}^{s_{1}} \ldots x_{n}^{s_{n}}$ tais que $s=\left(s_{1}, \ldots, s_{n}\right) \in C(\Delta)$. Isto é,

$$
\mathbb{C}[[\Delta]]=\left\{g_{\mathrm{C}(\Delta)}(x):=\sum_{\mathrm{LCC}(\Delta), \mathrm{Lfinito}} g_{\mathrm{L}} \quad: g \in \mathcal{O}_{n}\right\} .
$$

A filtração de Newton associada a estes anéis é a restrição de $\left\{\mathcal{A}_{q}\right\}$ a $\mathbb{C}[[\Delta]]$. Esta filtração tem a seguinte propriedade:

para todo $k \in C(\Delta)$, existe uma n-upla de inteiros $\left(a_{1}, \ldots, a_{n}\right)$ tal que $\Phi(6)^{k}=\sum_{i=1}^{n} a_{i} k_{i}$.

Definição 3.1.7. Dado um ideal I em $\mathcal{O}_{n}$, denotamos por $C(\bar{I})$ o fecho convexo em $\mathbb{R}^{n}+$ do conjunto $\left\{k \in \mathbb{N}^{n} \mid x^{k} \in \bar{I}\right\}$.

\subsection{Ideais monomiais}

Um ideal que pode ser gerado por monômios, é dito um ideal monomial. No decorrer desta seção, consideramos $I=\left(g_{1}, \ldots, g_{k}\right)$ um ideal de codimensão finita em ' $\mathcal{O}_{n}$ com

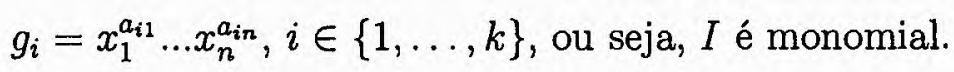


Destacamos aqui, alguns resultados a respeito de tais ideais, extraídos de [19], onde B. Teissier obtém uma expressão para a multiplicidade de um ideal monomial $I$ em função de um volume que está relacionado com $\Gamma_{+}(I)$.

Para chegar a essa expressão, Teissier utiliza o fecho integral de $I$, obtendo interessantes informações a respeito de fecho integral, poliedro de Newton e multiplicidade de ideais monomiais. Esses resultados contêm a essência do que nos propomos a fazer neste capítulo, que é relacionar fecho integral, multiplicidade e poliedro de Newton para a classe dos ideais Newton não-degenerados.

Teorema 3.2.1. [ B. Teissier [19], pag.129] Seja I um ideal monomial de codimensão finita em $\mathcal{O}_{n}$. Se $h \in \bar{I}$, então todos os monômios que aparecem na expressão de $h$ são representados em $\mathbb{R}^{n}$ por pontos situados em $\Gamma_{+}(I)$ ou seja, $\Gamma_{+}(h) \subset \Gamma_{+}(I)$.

Demonstração: Sejam $h=\sum a_{k} x^{k} \in \bar{I}$ e $b=\left(b_{1}, \ldots, b_{n}\right) \in \operatorname{supp}(h)$. Dada uma face $\Delta$ de $\Gamma_{+}(I)$, existem $\lambda_{1}, \ldots, \lambda_{n}, \eta \in \mathbb{R}$ tais que

$$
\Delta=\left\{\left(k_{1}, \ldots, k_{n}\right) \in \Gamma_{+}(I): \lambda_{1} k_{1}+\ldots+\lambda_{n} k_{n}=\eta\right\} .
$$

Seja $N=\lambda_{1} b_{1}+\ldots+\lambda_{n} b_{n}$. Mostraremos que $N \geq \eta$.

Dada uma curva analítica $\varphi(t)=\left(c_{1} t^{\lambda_{1}}, \ldots, c_{n} t^{\lambda_{n}}\right)$, temos $(h \circ \varphi)(t)=\left(a_{j_{1}} x^{\alpha_{1}}\left(c_{1}, \ldots, c_{n}\right)+\ldots+a_{j_{l}} x^{\alpha_{l}}\left(c_{1}, \ldots, c_{n}\right)\right) t^{N}+\sum_{m \neq N} q_{m}\left(c_{1}, \ldots, c_{n}\right) t^{m}=$ $\left(a_{j_{1}} c_{1}^{\alpha_{11}} \ldots c_{n}^{\alpha_{1 n}}+\ldots+a_{j_{l}} c_{1}^{\alpha_{l 1}} \ldots c_{n}^{\alpha_{l n}}\right) t^{N}+\sum_{m \neq N} q_{m}\left(c_{1}, \ldots, c_{n}\right) t^{m}$.

Assim como $q_{m}\left(c_{1}, \ldots, c_{n}\right), p\left(c_{1}, \ldots, c_{n}\right)=a_{j_{1}} c_{1}^{\alpha_{11}} \ldots c_{n}^{\alpha_{1 n}}+\ldots+a_{j_{l}} c_{1}^{\alpha_{l 1}} \ldots c_{n}^{\alpha_{l n}}$ é um polinômio em $c_{1}, \ldots, c_{n}$. Portanto, existem $d_{1}, \ldots, d_{n} \in \mathbb{R}$ tais que $p\left(d_{1}, \ldots, d_{n}\right) \neq 0$.

Consideremos então, $\psi:(\mathbb{C}, 0) \rightarrow\left(\mathbb{C}^{n}, 0\right)$ dada por $\psi(t)=\left(d_{1} t^{\lambda_{1}}, \ldots, d_{n} t^{\lambda_{n}}\right)$. Segue do que vimos acima e do teorema 2.3.6, que

$$
v\left(x^{b} \circ \psi\right) \geq v(h \circ \psi) \geq v(g \circ \psi), \quad \forall \quad g \in I
$$

pois, $h \in \bar{I}$. Em particular, a desigualdade é válida para um gerador $g_{i}(x)=x_{1}^{k_{1}} \ldots x_{n}^{k_{n}}$ de $I$ tal que $\left(k_{1}, \ldots, k_{n}\right) \in \Delta$. Assim,

$$
N=\lambda_{1} b_{1}+\ldots+\lambda_{n} b_{n}=v\left(x^{b} \circ \psi\right) \geq v\left(g_{i} \circ \psi\right)=\lambda_{1} k_{1}+\ldots+\lambda_{n} k_{n}=\eta .
$$


Isso significa que $b$ está acima da face $\Delta$. Como tomamos $\Delta$ arbitrariamente, obtemos $b \in \Gamma_{+}(I)$.

Proposição 3.2.2. [ B. Teissier, [19], pag.129] Seja I um ideal monomial de codimensão finita em $\mathcal{O}_{n}$. Se o ponto $b=\left(b_{1}, \ldots, b_{n}\right) \in \Gamma_{+}(I)$ representa o monômio $x^{b}=x_{1}^{b_{1}} \ldots x_{n}^{b_{n}}$, então $x^{b} \in \bar{I}$.

Demonstração: De fato, se $h=x_{1}{ }^{b_{1}} \ldots x_{n}{ }^{b_{n}} \notin \bar{I}$, então existe uma curva analítica

$$
\varphi:(\mathbb{C}, 0) \rightarrow\left(\mathbb{C}^{n}, 0\right) ; \quad \varphi=\left(\varphi_{1}, \ldots, \varphi_{n}\right)
$$

tal que $v(h \circ \varphi)<v\left(g_{j} \circ \varphi\right), \quad \forall j \in\{1, \ldots, k\}$, em que $\left\{g_{1}, \ldots, g_{k}\right\}$ é um conjunto de geradores de $I$ da forma $g_{j}=x_{1}{ }^{a_{j 1}} \ldots x_{n}{ }^{a_{j n}}$. Sendo $\varphi_{i}(t)=t^{s_{i}}+\operatorname{ord}\left(s_{i}\right), \operatorname{com} \operatorname{ord}\left(s_{i}\right)$ denotando os termos de ordem superior a $s_{i}$, temos

$$
\begin{gathered}
v(h \circ \varphi)=v\left(\varphi_{1}^{b_{1}} \ldots \varphi_{n}^{b_{n}}\right)=s_{1} b_{1}+\ldots+s_{n} b_{n}, \\
v\left(g_{j} \circ \varphi\right)=v\left(\varphi_{1}^{a_{j 1}} \ldots \varphi_{n}^{a_{j n}}\right)=s_{1} a_{j 1}+\ldots+s_{n} a_{j n} .
\end{gathered}
$$

Chamando $\Lambda_{i}$ o hiperplano dado por

$$
s_{1} z_{1}+\ldots+s_{n} z_{n}=\eta_{i}
$$

obtemos $v(h \circ \varphi)<v\left(g_{j} \circ \varphi\right)$, logo $b=\left(b_{1}, \ldots, b_{n}\right)$ encontra-se em um hiperplano situado abaixo do hiperplano $\Lambda_{j}$ ao qual $a_{j}=\left(a_{j 1}, \ldots, a_{j n}\right)$ pertence. Como $\Gamma_{+}(I)$ é determinado por $a_{1}, \ldots, a_{k}$, o primeiro hiperplano $\Lambda_{s}$ que intersecta $\Gamma_{+}(I)$ deve conter algum $a_{j}$; isto é, $\Lambda_{s}=\Lambda_{j}$ para algum $j \in\{1, \ldots, k\}$. Como

$$
b \in \Lambda_{v(h \circ \varphi)} \text { e } \Lambda_{v(h \circ \varphi)} \cap \Lambda_{j}=\varnothing
$$

$\Lambda_{v(h \circ \varphi)}$ não intersecta $\Gamma_{+}(I)$ e, portanto, $b \notin \Gamma_{+}(I)$.

Teorema 3.2.3. [ B. Teissier, [19], pag. 129, 130] Se I é um ideal monomial de codimensão finita em $\mathcal{O}_{n}$, então $\bar{I}=\left(\left\{x^{k}: k \in \Gamma_{+}(I)\right\}\right)$. Ou seja, $C(\bar{I})=\Gamma_{+}(I)$.

Demonstração: Seja $\left\{g_{1}, \ldots, g_{k}\right\}$ um conjunto de geradores de $I$ com

$$
g_{j}=x_{1}{ }^{a_{j 1}} \ldots x_{n}{ }^{a_{j n}}, \quad j \in\{1, \ldots, k\} .
$$


Sendo $\left(\Gamma_{+}(I) \backslash E(I)\right) \cap \mathbb{Z}^{n}$ finito, seja $\left\{\beta_{1}, \ldots, \beta_{d}\right\}=\left(\Gamma_{+}(I) \backslash E(I)\right) \cap \mathbb{Z}^{n}$. Considere $J$ o ideal de $\mathcal{O}_{n}$ gerado por

$$
\left\{x^{s}: s \in\left\{a_{1}, \ldots, a_{k}, \beta_{1}, \ldots, \beta_{d}\right\}\right\}
$$

em que $a_{j}=\left(a_{j 1}, \ldots, a_{j n}\right)$ representa o gerador $g_{j}$ de $I$. Mostraremos que $J=\bar{I}$.

Dado $x^{s} \in J$, temos $s \in \Gamma_{+}(I)$. Segue da proposição 3.2 .2 que $x^{s} \in \bar{I}$, logo $J \subset \bar{I}$.

Suponhamos, por absurdo, que exista $h \in \bar{I} \backslash J$. Podemos escrever $h(x)=$ $x^{m_{0}} \sum_{m=m_{0}}^{\infty} c_{m} x^{m-m_{0}}$ com $c_{m_{0}} \neq 0$. Como $h \notin J, \quad x^{m_{0}}$ não é múltiplo de $x^{a_{j}}$ ou de $x^{\beta_{i}}$ para quaisquer $j \in\{1, \ldots, k\}, i \in\{1, \ldots, d\}$. Escrevamos $\beta_{j}=\left(\beta_{j 1}, \ldots, \beta_{j n}\right)$ e $m_{0}=\left(m_{01}, \ldots, m_{0 n}\right)$. Então, para cada $j \in\{1, \ldots, k\}, i \in\{1, \ldots, d\}$, existem $s, l \in\{1, \ldots, n\}$ tais que $m_{0 s}<a_{j s}$ e $m_{0 l}<\beta_{i l}$. Mas, $m_{0 s}<a_{j s}$ implica em $m_{0 s} \notin a_{j}+\mathbb{R}^{n}{ }_{+}$. Como isso ocorre para qualquer $j \in\{1, \ldots, k\}, \quad m_{0} \notin E(I)$. Por outro lado, segue de $m_{0 l}<\beta_{i l}$ que $m_{0} \neq \beta_{i}$. Uma vez que isso ocorre para qualquer $i \in\{1, \ldots, n\}$ e $m_{0} \in \mathbb{Z}^{n}$, concluímos que $m_{0} \notin \Gamma_{+}(I)$. Como $h \in \bar{I}$, entramos em contradição com o Teorema 3.2.1. Portanto, $\left.\bar{I}=\left(\left\{x^{s}: s \in\left\{a_{1}, \ldots, a_{k}, \beta_{1}, \ldots, \beta_{d}\right)\right\}\right\}\right)=\left(\left\{x^{s}: s \in \Gamma_{+}(I)\right\}\right)$.

Geometricamente, esse teorema nos diz que no caso em que $I$ é monomial, a diferença entre $\bar{I}$ e $I$ é dada pelos pontos situados acima do poliedro de Newton de $I$ e abaixo de $E(I)$.

Dado um ideal $I$ de codimensão finita em $\mathcal{O}_{n}$, denotamos por $I_{0}$ o ideal de $\mathcal{O}_{n}$ gerado por $\left\{x^{s}: s \in \Gamma_{+}(I)\right\}$. Claramente vemos que $I_{0}$ tem codimensão finita em $\mathcal{O}_{n}$ e, $\Gamma_{+}(I)=\Gamma_{+}\left(I_{0}\right)$.

Como conseqüência imediata dos resultados vistos temos o seguinte

Corolário 3.2.4. Dado um ideal monomial I de codimensão finita $\mathrm{em} \mathcal{O}_{n}$, temos:

(i) $C(\bar{I})=\Gamma_{+}(I)$.

(ii) $\overline{I_{0}}=I_{0}$.

(iii) $\Gamma_{+}(\bar{I})=\Gamma_{+}(I)$. 
Demonstração: De fato, (i) segue diretamente do Teorema 3.2.1 e da proposição 3.2.2.

(ii) Sendo $I_{0}$ monomial, segue do Teorema 3.2.3 que

$$
\overline{I_{0}}=\left(\left\{x^{s}: s \in \Gamma_{+}\left(I_{0}\right)\right\}\right)=\left(\left\{x^{s}: s \in \Gamma_{+}(I)\right\}\right)=I_{0} .
$$

(iii) Segue do Teorema 3.2.3 que $\bar{I}=\left(\left\{x^{s}: s \in \Gamma_{+}(I)\right\}\right)$. Então,

$$
E(\bar{I})=\bigcup_{s \in \Gamma_{+}(I) \cap \mathbb{Z}^{n}} \mathrm{~s}+\mathbb{R}_{+}^{\mathrm{n}} \subset \Gamma_{+}(I) \text {. Daí, } \Gamma_{+}(\bar{I}) \subset \Gamma_{+}(I) . \mathrm{e}, \Gamma_{+}(\bar{I})=\Gamma_{+}(I) \text {. }
$$

Lema 3.2.5. Se $I$ é um ideal de codimensão finita $\mathrm{em} \mathcal{O}_{n}$, não necessariamente monomial, então $\Gamma_{+}(\bar{I})=\Gamma_{+}(I)$.

Demonstração: De fato, sendo $I_{0}=\left(\left\{x^{s}: s \in \Gamma_{+}(I)\right\}\right)$, segue do item (ii) do corolário acima que $\bar{I} \subset \overline{I_{0}}=I_{0}$. Logo, $I \subset \bar{I} \subset I_{0} \mathrm{e}$,

$$
\Gamma_{+}(I) \subset \Gamma_{+}(\bar{I}) \subset \Gamma_{+}\left(I_{0}\right)=\Gamma_{+}(I)
$$

Teorema 3.2.6. [B. Teissier, [19], pag.131] Se I é um ideal monomial de codimensão finita em $\mathcal{O}_{n}$, então $e(I)=n ! v(I)$, em que $v(I)$ é o volume $n$-dimensional do conjunto $\mathbb{R}_{+}^{n} \backslash \Gamma_{+}(I)$.

Lema 3.2.7. Se I é um ideal monomial de codimensão finita em $\mathcal{O}_{n}$, então

$$
\lim _{s \rightarrow \infty} \frac{1}{s} E\left(I^{s}\right)=\Gamma_{+}(I) .
$$

Caso 1: $n=2, I=\left(x^{m_{1}}, y^{m_{2}}\right)$ Neste caso, o poliedro de Newton de $I$ possui apenas uma face compacta $\Delta$ de dimensão 1 .

Dado $(a, b) \in \Gamma_{+}(I) \backslash \Delta$, queremos mostrar que existe $s \in \mathbb{N}$ tal que $(a, b) \in \frac{1}{s} E\left(I^{s}\right)$. Se $b \geq \beta$ ou $a \geq m_{1}$, então $(a, b) \in E(I)$. Suponhamos então, que $b<m_{2}$ e $a<m_{1}$, ou seja, $(a, b) \in \Gamma_{+}(I) \backslash(E(I) \cup \Delta)$. Consideremos os pontos $p, q$ pertencentes, respectivamente, a $\Delta \cap[(a, b),(a, 0)], \Delta \cap[(a, b),(0, b)]$. Segue da densidade dos racionais em $\mathbb{R}$ que existe $t \in \mathbb{Q}$ tal que o ponto $t\left(m_{1}, 0\right)+(1-t)\left(0, m_{2}\right) \in[p, q]$. 
Como $t=\frac{k}{s}$, tomemos $k_{1}=k$ e $k_{2}=s-k$. Então, $g=x^{m_{1} k_{1}} y^{m_{2} k_{2}} \in I^{s}$. Daí, $\left(m_{1} k_{1}, m_{2} k_{2}\right) \in E\left(I^{s}\right)$ e $\left(\frac{k_{1}}{s} m_{1}, \frac{k_{2}}{s} m_{2}\right) \in \frac{1}{s} E\left(I^{s}\right)$. Como $\left(\frac{k_{1}}{s} m_{1}, \frac{k_{2}}{s} m_{2}\right)+\mathbb{R}_{+}^{2} \subset \frac{1}{s} E\left(I^{s}\right)$, $(a, b) \in \frac{1}{s} E\left(I^{s}\right)$. Logo, $\Gamma_{+}(I) \subset \lim _{s \rightarrow \infty} \frac{1}{s} E\left(I^{s}\right)$.

Caso 2: Para qualquer ideal monomial de codimensão finita $\mathrm{em} \mathcal{O}_{n}$ do tipo $I=\left(x_{1}^{\alpha_{1}}, \ldots, x_{n}^{\alpha_{n}}\right)$, isto é, quando $\Gamma_{+}(I)$ possui apenas uma face compacta $\Delta$ de dimensão $n-1$, procedemos da mesma maneira.

Novamente, precisamos analisar apenas os pontos $\left(a_{1}, \ldots, a_{n}\right) \in \Gamma_{+}(I) \backslash(E(I) \cup \Delta)$. Consideramos os pontos $p_{1}, \ldots, p_{n}$ tais que $p_{i} \in\left[\left(a_{1}, \ldots, a_{n}\right),\left(0, \ldots, a_{i}, \ldots, 0\right)\right] \cap \Delta, i \in$ $\{1, \ldots, n\}$. Observamos que $\varphi: \mathbb{R}^{n-1} \rightarrow \mathbb{R}^{n}$ dada por

$$
\varphi\left(t_{1}, \ldots, t_{n-1}\right)=\left(t_{1} \alpha_{1}, \ldots, t_{n-1} \alpha_{n-1},\left(1-t_{1}-\ldots-t_{n-1}\right) \alpha_{n}\right)
$$

é uma parametrização para $\Delta$. Usando a densidade de $\mathbb{Q}^{n-1}$ em $\mathbb{R}^{n-1}$, tomamos $\left(l_{1}, \ldots, l_{n-1}\right) \in \mathbb{Q}^{n-1}$ tal que $\varphi\left(l_{1}, \ldots, l_{n-1}\right)$ pertence ao poliedro determinado por $p_{1}, \ldots, p_{n}$. Sendo $l_{1}=\frac{r_{1}}{s_{1}}, \ldots, l_{n-1}=\frac{r_{n-1}}{s_{n-1}}$, tomamos $s=s_{1} \ldots s_{n-1}, k_{i}=\frac{r_{i} s}{s_{i}}, i \in\{1, \ldots, n-1\} \mathrm{e}$ $k_{n}=s-\left(k_{1}+\ldots+k_{n-1}\right)$. Assim, $\varphi\left(\frac{k_{1}}{s}, \ldots, \frac{k_{n-1}}{s}\right)=\left(\frac{k_{1}}{s} m_{1}, \frac{k_{2}}{s} m_{2}, \ldots, \frac{k_{n}}{s} m_{n}\right)$. Além disso, $g=x_{1}^{m_{1} k_{1}} \ldots x_{n}^{m_{n} k_{n}} \in I^{s}$ pois $k_{1}+\ldots+k_{n}=s$. Daí, $\left(m_{1} k_{1}, \ldots, m_{n} k_{n}\right) \in E\left(I^{s}\right) \mathrm{e}$, $\left(\frac{k_{1}}{s} m_{1}, \frac{k_{2}}{s} m_{2}, \ldots, \frac{k_{n}}{s} m_{n}\right) \in \frac{1}{s} E\left(I^{s}\right)$. Como $\left(\frac{k_{1}}{s} m_{1}, \frac{k_{2}}{s} m_{2}, \ldots, \frac{k_{n}}{s} m_{n}\right)$ pertence ao poliedro determinado por $p_{1}, \ldots, p_{n},\left(a_{1}, \ldots, a_{n}\right) \in\left(\frac{k_{1}}{s} m_{1}, \frac{k_{2}}{s} m_{2}, \ldots, \frac{k_{n}}{s} m_{n}\right)+\mathbb{R}_{+}^{n} \subset \frac{1}{s} E\left(I^{s}\right)$. Portanto, $\Gamma_{+}(I) \subset \lim _{s \rightarrow \infty} \frac{1}{s} E\left(I^{s}\right)$.

Caso geral: Agora, mostrar que $\Gamma_{+}(I) \subset \lim _{s \rightarrow \infty} \frac{1}{s} E\left(I^{s}\right)$ para um ideal monomial $I$ de $\mathcal{O}_{n}$ cujo poliedro de Newton possui mais de uma face compacta, tornou-se uma tarefa fácil. Novamente, estudamos apenas os pontos de $\Gamma_{+}(I) \backslash(\Gamma(I) \cup E(I))$. Dado $p \in \Gamma_{+}(I) \backslash(\Gamma(I) \cup E(I))$, tomamos $\Delta$ uma face compacta de $\Gamma_{+}(I)$ de dimensão $n-1$ tal que $p \in C(\Delta)$. Repetindo o procedimento feito feito acima, encontramos $s \in \mathbb{N}$ tal que $p \in \frac{1}{s} E\left(I^{s}\right)$. Logo, $\Gamma_{+}(I) \subset \lim _{s \rightarrow \infty} \frac{1}{s} E\left(I^{s}\right)$.

Para mostrar a inclusão contrária, consideramos $\left\{g_{1}, \ldots, g_{m}\right\}$ um conjunto de geradores de $I$ da forma $g_{i}=x_{1}^{k_{i 1}} \ldots x_{n}^{k_{i n}}$. Então, para qualquer $s \in \mathbb{N}$, temos

$$
E\left(I^{s}\right)=\left\{\left(\alpha_{1} k_{i 1}, \ldots, \alpha_{n} k_{i n}\right)+v: \alpha_{1}+\ldots+\alpha_{n}=s, v \in \mathbb{R}_{+}^{n}, i \in\{1, \ldots, m\}\right\} .
$$


Daí,

$$
\frac{1}{s} E\left(I^{s}\right)=\left\{\left(\frac{\alpha_{1}}{s} k_{i 1}, \ldots, \frac{\alpha_{n}}{s} k_{i n}\right)+v: \alpha_{1}+\ldots+\alpha_{n}=s, v \in \mathbb{R}_{+}^{n}, i \in\{1, \ldots, m\}\right\} .
$$

Assim, dado $p \in \frac{1}{s} E\left(I^{s}\right)$, temos $p=\left(\frac{\alpha_{1}}{s} k_{i 1}, \ldots, \frac{\alpha_{n}}{s} k_{\text {in }}\right)+v \operatorname{com} \frac{\alpha_{1}}{s}+\ldots+\frac{\alpha_{n}}{s}=1$. Logo, $p-v$ é uma combinação linear convexa de elementos de $E(I)$; portanto, $p-v \in$ $\Gamma_{+}(I), p \in \Gamma_{+}(I)$ e, $\frac{1}{s} E\left(I^{s}\right) \subset \Gamma_{+}(I), \quad \forall s \in \mathbb{N}$.

Dado um ponto $p \in \lim _{s \rightarrow \infty} \frac{1}{s} E\left(I^{s}\right)$, existe uma seqüência no conjunto $\bigcup_{s \in \mathbb{N}} \frac{1}{s} E\left(I^{s}\right)$ convergindo para $p$. Sendo esta, uma seqüência em $\Gamma_{+}(I)$ e $\Gamma_{+}(I)$ fechado, $p \in \Gamma_{+}(I)$. Portanto, $\lim _{s \rightarrow \infty} \frac{1}{s} E\left(I^{s}\right) \subset \Gamma_{+}(I)$ e, está mostrado o Lema 3.2.7.

Observação 3.2.8. Dado um ideal $J, \mathbb{R}_{+}^{n} \backslash E(J)$ é união finita de cubos $n-1$ dimensionais. Mais precisamente, $\mathbb{R}_{+}^{n} \backslash E(J)=\bigcup_{i=1}^{m}\left[a_{i 1}, b_{i 1}\right) \times\left[a_{i 2}, b_{i 2}\right) \times \ldots \times\left[a_{i n}, b_{i n}\right)$. Então, $\operatorname{vol}\left(\mathbb{R}_{+}^{n} \backslash E(J)\right)=\sum_{i=1}^{m} \operatorname{vol}\left(\left[a_{i 1}, b_{i 1}\right) \times\left[a_{i 2}, b_{i 2}\right) \times \ldots \times\left[a_{i n}, b_{i n}\right)\right)$. Mas, $\operatorname{vol}\left(\left[a_{i 1}, b_{i 1}\right) \times\right.$ $\left.\left[a_{i 2}, b_{i 2}\right) \times \ldots \times\left[a_{i n}, b_{i n}\right)\right)$ é igual ao número de pontos com coordenandas inteiras que este bloco contém. Como $\mathbb{R}_{+}^{n} \backslash E(J)$ é união disjunta de blocos deste tipo, $\operatorname{vol}\left(\mathbb{R}_{+}^{n} \backslash E(J)\right)$ é igual ao número de pontos com coordenadas inteiras contidos em $\mathbb{R}_{+}^{n} \backslash E(J)$.

Demonstração do Teorema 3.2.6: Seja $A=\mathbb{C}\left\{x_{1}, \ldots, x_{n}\right\}$. Então, $\operatorname{dim}_{\mathbb{C}} \frac{A}{I}$ é igual ao número de pontos com coordenadas inteiras em $\mathbb{R}_{+}^{n} \backslash E(I)$. Assim,

$$
\operatorname{dim}_{\mathbb{C}} \frac{A}{I^{s}}=\operatorname{card}\left\{\mathbb{N}^{n} \backslash E\left(I^{s}\right)\right\} .
$$

Segue do lema 3.2.7 e da observação feita acima que

Assim, $\quad e(I)=\lim _{s \rightarrow \infty} \frac{n !}{s^{n}} \operatorname{dim}_{\mathbb{C}} \frac{A}{I^{s}}=n ! v(I)$.

$$
\begin{aligned}
\mathrm{v}(I) & =\operatorname{vol}\left(\mathbb{R}_{+}^{n} \backslash \Gamma_{+}(I)\right)=\operatorname{vol}\left(\mathbb{R}_{+}^{n} \backslash \lim _{s \rightarrow \infty} \frac{1}{s} E\left(I^{s}\right)\right) \\
& =\lim _{s \rightarrow \infty} \operatorname{vol}\left(\mathbb{R}_{+}^{n} \backslash \frac{1}{s} E\left(I^{s}\right)\right)=\lim _{s \rightarrow \infty} \frac{1}{s^{n}} \operatorname{vol}\left(\mathbb{R}_{+}^{n} \backslash E\left(I^{s}\right)\right) \\
& =\lim _{s \rightarrow \infty} \frac{1}{s^{n}} \operatorname{card}\left\{\mathbb{N}^{n} \backslash E\left(I^{s}\right)\right\}=\lim _{s \rightarrow \infty} \frac{1}{s^{n}} \operatorname{dim}_{\mathbb{C}} \frac{A}{I^{s}} .
\end{aligned}
$$


Observação 3.2.9. Se I, J são ideais monomiais de codimensão finita em $\mathcal{O}_{n}$, então $E(I J)=E(I)+E(J)$.

\subsection{Caracterizando ideais Newton não-degenerados}

Nesta seção, trabalhamos com ideais Newton não-degenerados e generalizamos os resultados apresentados na seção anterior para esta classe de ideais. Introduzamos então, o conceito de Newton não-degeneração.

No que segue, consideramos $I=\left(g_{1}, \ldots, g_{k}\right)$ um ideal tal que $\operatorname{dim}_{\mathbb{C}} \frac{\mathcal{O}_{n}}{I}$ é finita.

Definição 3.3.1. Uma face compacta $\Delta \in \Gamma(I)$ é Newton não-degenerada se o ideal gerado por $\left\{g_{1}, \ldots, g_{k \Delta}\right\}$ tem codimensão finita em $\mathbb{C}[[\Delta]]$.

Definição 3.3.2. Um ideal I é Newton não-degenerado se todas as faces compactas são Newton não-degeneradas. Quando o ideal não é Newton não-degenerado, é dito Newton degenerado.

A definição anterior é equivalente [ver Kouchnirenko [11], item 1.19] ao seguinte: $I$ é Newton não-degenerado se, e somente se, para cada face compacta $\Delta \in \Gamma(I)$, as equações

$$
g_{1 \Delta}(x)=g_{2 \Delta}(x)=\ldots=g_{k_{\Delta}}(x)=0
$$

não possuem solução comum em $(\mathbb{C} \backslash\{0\})^{n}$.

Claramente vemos que $I_{0}=\left(\left\{x^{s}: s \in \Gamma_{+}(I)\right\}\right)$ é Newton não-degenerado e $\Gamma_{+}\left(I_{0}\right)=\Gamma_{+}(I)$. É interessante observar que dois ideais podem ter o mesmo poliedro de Newton e apenas um deles ser Newton não-degenerado. Por exemplo,

$$
I=\left(g_{1}, g_{2}\right) \quad \text { com } \quad g_{1}=x^{8}+y^{8} \quad \text { e } \quad g_{2}=x y^{5}-x^{5} y
$$

é Newton degenerado pois

$$
g_{1 \Delta_{2}}(t, t)=g_{2_{\Delta_{2}}}(t, t)=0 \quad \forall t \neq 0
$$

em que $\Delta_{2}$ é a face compacta com vértices $(1,5),(5,1)$. Por outro lado,

$$
I_{0}=\left(\left\{x^{8}, y^{8}, x^{1} y^{5}, x^{5} y^{1}, x^{2} y^{4}, x^{3} y^{4}, x^{4} y^{3}, x^{4} y^{4}\right\}\right)
$$


é Newton não-degenerado embora $\Gamma_{+}(I)=\Gamma_{+}\left(I_{0}\right)$.

Observamos que por $I \subset I_{0}$ e $\Gamma_{+}(I)=\Gamma_{+}\left(I_{0}\right)$, temos $e(I) \geq e\left(I_{0}\right)=n ! v\left(I_{0}\right)=$ $n ! v(I)$. Mostramos nesta seção que vale a igualdade apenas quando $I$ é Newton não-degenerado.

Lema 3.3.3 ( M.J.Saia, $[14]) . C(\bar{I}) \subset \Gamma_{+}(I)$.

Demonstração: De fato, se $m \notin \Gamma_{+}(I)$, então $f i l\left(x^{m}\right)<f i l\left(g_{i}\right)$ para cada gerador $g_{i}$ de $I$. Seja $\Delta$ uma face compacta dedimensão $(n-1)$ de $\Gamma_{+}(I)$ tal que $m \in C(\Delta)$ e, consideremos $\psi(t)=\left(t^{a_{1}}, \ldots, t^{a_{n}}\right)$, em que $\left(a_{1}, \ldots, a_{n}\right)$ é a n-upla de inteiros que define a restrição a $\mathbb{C}[[\Delta]]$ da função linear por partes $\Phi$ associada a $\Gamma_{+}(I)$.

Então, $v\left(x^{m} \circ \psi\right)<v\left(g_{i} \circ \psi\right) \quad \forall i \in\{1, \ldots, k\}$, em que $v$ denota a valoração complexa. Aplicando o critério avaliativo do Teorema 2.3.6 para a curva $\psi$, obtemos que $x^{m} \notin \bar{I}$. Logo, $m \notin C(\bar{I})$.

A igualdade desses conjuntos ocorre apenas quando $I$ é Newton não-degenerado. Nosso objetivo é mostrar esse resultado.

Nesse momento, convém introduzirmos a noção de redução de um ideal.

Definição 3.3.4. Dados dois ideais $J \subset I$, dizemos que $J$ é uma redução de $I$ quando existe $r>0$ tal que $I^{r+1}=J I^{r}$.

Um resultado que caracteriza as reduções de um ideal em termos do fecho integral é o seguinte

Teorema 3.3.5 (Rees, [19], pag. 132). Sejam $I \subset J$ ideais de codimensão finita em $\mathcal{O}_{n}$. As seguintes condições são equivalentes:

(i) J é uma redução de I;

(ii) $e(I)=e(J)$;

(iii) $\bar{I}=\bar{J}$.

Lema 3.3.6. Seja I um ideal de codimensão finita em $\mathcal{O}_{n}$. Se $I$ é Newton nãodegenerado, então $\bar{I}=I_{0}$. 
Demonstração: Como $I \subset I_{0}$, segue do item (ii) do Corolário 3.2.4 que $\bar{I} \subset \overline{I_{0}}=I_{0}$. Suponhamos, por absurdo, que exista um monômio $h=x_{1}{ }^{k_{1}} \ldots x_{n}{ }^{k_{n}} \in I_{0} \backslash \bar{I}$. Então, segue do Teorema 2.3.6 que existe uma curva analítica $\varphi:(\mathbb{C}, 0) \rightarrow\left(\mathbb{C}^{n}, 0\right)$ tal que

$$
h \circ \varphi \notin\left(\varphi^{*}(I)\right) \mathcal{O}_{1} ; \quad \text { ou seja, } \quad v(h \circ \varphi)<v(g \circ \varphi), \quad \forall \quad g \in I,
$$

em que $v$ é a valoração natural dos complexos. Escrevamos

$$
\varphi(t)=\left(\varphi_{1}(t), \ldots, \varphi_{n}(t)\right), \quad \varphi_{i}(t)=a_{i} t^{m_{i}}+\operatorname{ord}\left(m_{i}\right), \quad a_{i} \neq 0 \quad i \in\{1, \ldots, n\} .
$$

Sem perda de generalidade, podemos supor que nenhuma das funções coordenadas de $\varphi$ é identicamente nula. De fato, se $\varphi_{i}=0$ para algum $i \in\{1, \ldots, n\}$, então $k_{i}=0$ pois do contrário, teríamos $h \circ \varphi=\varphi_{1}{ }^{k_{1}} \ldots \varphi_{i}{ }^{k_{i}} \ldots \varphi_{n}{ }^{k_{n}}=0 \in\left(\varphi^{*}(I)\right) \mathcal{O}_{1}$ o que já sabemos que não ocorre. Nesse caso, sendo

$$
h=x_{1}^{k_{1}} \ldots x_{i-1}^{k_{i-1}} x_{i+1}^{k_{i+1}} \ldots x_{n}^{k_{n}}
$$

substituímos $\varphi$ por $\tilde{\varphi}=\left(\tilde{\varphi}_{1}, \ldots, \tilde{\varphi}_{i}, \ldots, \tilde{\varphi_{n}}\right)$ com $\tilde{\varphi}_{j}=\varphi_{j}, j \neq i$ e $\tilde{\varphi}_{i}(t)=t$ pois $h \circ \varphi=h \circ \tilde{\varphi}$.

Seja $d_{0}=k_{1} m_{1}+\ldots+k_{n} m_{n}=v(h \circ \varphi)$. Então, $d_{0}<v(g \circ \varphi) \quad \forall g \in I$. Consideremos os hiperplanos $\Lambda_{j}$ dados por

$$
\alpha_{j 1} m_{1}+\ldots+\alpha_{j n} m_{n}=d_{j}, \quad j \in \mathbb{N} .
$$

Seja $s$ tal que $\Lambda_{s}$ é o primeiro desses hiperplanos que intersecta $\Gamma_{+}(I)$; isto é, $s$ é tal que

$$
d_{s}=\min \left\{d_{j}: \Lambda_{j} \cap \Gamma_{+}(I) \neq \varnothing\right\} .
$$

Sejam $\Delta$ a face de dimensão $n-1$ de $\Gamma_{+}(I)$ tocada por $\Lambda_{s}$ e, $\Delta_{1} \subset \Delta$ uma face compacta de $\Gamma_{+}(I)$.

Dado $g \in I$, temos $g=b_{i_{0}} x_{1}{ }^{i_{01}} x_{2}{ }^{i_{02}} \ldots x_{n}{ }^{i_{0 n}}+\operatorname{ord}\left(i_{01}+i_{02}+\ldots+i_{0 n}\right)$ e $g \circ \varphi=$ $a b_{i_{0}} t^{i_{01} m_{1}+\ldots+i_{0 n} m_{n}}+\operatorname{ord}\left(i_{01} m_{1}+\ldots+i_{0 n} m_{n}\right)$ Afirmamos que $g_{\Delta_{1}} \circ \varphi=0$. De fato, do contrário, teríamos: $\quad g_{\Delta_{1}}(x)=b_{i_{1}} x_{1}{ }^{i_{11}} x_{2}{ }^{i_{12}} \ldots x_{n}{ }^{i_{1 n}}+\operatorname{ord}\left(i_{11}+i_{12}+\ldots+i_{1 n}\right)$, $g_{\Delta_{1}} \circ \varphi=a b_{i_{0}} t^{i_{11} m_{1}+\ldots+i_{1 n} m_{n}}+\operatorname{ord}\left(i_{11} m_{1}+\ldots+i_{1 n} m_{n}\right) \operatorname{com} b_{i_{1}} \neq 0$ e,

$$
v\left(g_{\Delta_{1}} \circ \varphi\right)=i_{11} m_{1}+\ldots+i_{1 n} m_{n}=d_{s} \leq d_{0}
$$


Então, $v(g \circ \varphi) \leq v\left(g_{\Delta_{1}} \circ \varphi\right) \leq d_{0} \circ$ que contradiz $\circ$ fato de $v(g \circ \varphi)>v(h \circ \varphi)=d_{0}$ $\forall g \in I$.

Mas, $g_{\Delta_{1}} \circ \varphi \equiv 0$ para qualquer $g \in I$ e $\operatorname{Im} \varphi \nsubseteq(\mathbb{C} \backslash\{0\})^{n}$ contradiz a Newton não-degeneração de $I$. Assim, $h \circ \varphi \in\left(\varphi^{*}(I)\right) \mathcal{O}_{1}$ para qualquer curva analítica $\varphi:(\mathbb{C}, 0) \longrightarrow\left(\mathbb{C}^{n}, 0\right)$. Portanto, $h \in \bar{I}$ e $I_{0} \subset \bar{I}$.

Lema 3.3.7. Sejam I um ideal de codimensão finita em $\mathcal{O}_{n}$ e $J$ uma redução de $I$. Se I é Newton não-degenerado, então $J$ também é Newton não-degenerado.

Demonstração: Sem perda de generalidade, podemos supor que $I=\left(g_{1}, \ldots, g_{k}\right)$ é monomial. De fato, se I não for monomial, decorre do Teorema 3.3.5 e do Lema 3.3.6 que $\bar{J}=\bar{I}=I_{0}=\bar{I}_{0}$ já que $J$ é redução de $I$ e $I$ é Newton não-degenerado. Assim, $J$ é redução de $I_{0}$ e podemos trabalhar com $I_{0}$ ao invés de $I$.

Afirmamos que $I^{s}$ é Newton não-degenerado para qualquer $s \in \mathbb{N}$. Com efeito, se $I^{s}$ é Newton degenerado, então existem $\Delta \in \Gamma\left(I^{s}\right)$ e $x \in(\mathbb{C} \backslash\{0\})^{n}$ tais que

$$
h_{\Delta}(x)=0 \quad \forall h \in\left\{g_{1}^{\alpha_{1}} \ldots g_{k}^{\alpha_{k}} \quad: \quad \alpha_{1}+\ldots+\alpha_{k}=s\right\}
$$

Em particular, $g_{i \Delta}^{s}(x)=0$ para qualquer $i \in\{1, \ldots, k\}$. Como $I$ é monomial, obtemos que $\Delta^{\prime}=\frac{1}{s} \Delta$ é uma face compacta de $\Gamma_{+}(I)$. Assim, $g_{i \Delta^{\prime}}(x)=g_{i}{ }^{s}(x)=0 \quad \forall \quad i \in$ $\{1, \ldots, k\}$. Mas, isso contradiz o fato de $I$ ser Newton não-degenerado.

Lembramos que por $J$ ser redução de $I$, temos

$$
\Gamma_{+}(J)=\Gamma_{+}(\bar{J})=\Gamma_{+}(\bar{I})=\Gamma_{+}(I) \text {. }
$$

Além disso, existe $r>0$ tal que $I^{r}=J I^{r-1} \subset J$.

Seja $\Delta$ uma face compacta de $\Gamma_{+}(J)$. Então, segue da Observação 3.2 .9 que $r \Delta \in \Gamma\left(I^{r}\right)$ e, portanto, $I_{r \Delta}^{r} \subset J_{r \Delta} \subset J_{r \Delta}+J_{\Delta}$. Observamos que $I_{r \Delta}^{r}, J_{\Delta}, J_{r \Delta}+J_{\Delta}$ são subespaços vetoriais de $\mathbb{C}[[\Delta]]=\mathbb{C}[[r \Delta]]:=A$. Daí,

$$
\operatorname{dim} \frac{A}{J_{r \Delta}+J_{\Delta}} \leq \operatorname{dim} \frac{A}{I_{r \Delta}^{r}}<\infty
$$

por $I^{r}$ ser Newton não-degenerado. Portanto,

$$
\operatorname{dim} \frac{A}{J_{\Delta}} \leq \operatorname{dim} \frac{A}{J_{r \Delta}+J_{\Delta}}+\operatorname{dim} J_{r \Delta}<\infty,
$$

pois $\operatorname{dim} J_{r \Delta}<\infty$. Concluímos, assim, que $J$ é Newton não-degenerado. 
Teorema 3.3.8. Seja $I$ um ideal de codimensão finita em $\mathcal{O}_{n}$. Então, $I$ é Newton não-degenerado se, e somente se, $\bar{I}$ é monomial.

Demonstração: Se $I$ é Newton não-degenerado, segue do lema 3.3.6 que $\bar{I}$ é monomial. Reciprocamente, se $\bar{I}$ é monomial, então é Newton não-degenerado. Sendo $I$ uma redução de $\bar{I}$, segue do lema 3.3.7 que $I$ é Newton não-degenerado.

Proposição 3.3.9. Seja I um ideal de codimensão finita em $\mathcal{O}_{n}$. Então, I é Newton não-degenerado se, e somente se, $e(I)=n ! v(I)$.

Demonstração: Se $I$ é Newton não-degenerado, então segue do Lema 3.3.6 que $\bar{I}=I_{0}=\overline{I_{0}}$. Assim, $I$ é uma redução de $I_{0} ;$ logo,

$$
e(I)=e\left(I_{0}\right)=n ! v\left(I_{0}\right)=n ! v(I)
$$

pois $I_{0}$ é monomial e $\Gamma_{+}\left(I_{0}\right)=\Gamma_{+}(I)$. Reciprocamente, se $e(I)=n ! v(I)$, então decorre do Lema 3.2.6 e do fato de $\Gamma_{+}\left(I_{0}\right)=\Gamma_{+}(I)$ que $e(I)=e\left(I_{0}\right)$. Portanto, $I$ é uma redução de $I_{0}$. Sendo $I_{0}$ Newton não-degenerado, segue do Lema 3.3.7 que $I$ é Newton não degenerado.

Proposição 3.3.10. Seja I um ideal de codimensão finita em $\mathcal{O}_{n}$. Então, $e(I)=n ! v(I)$ se, e somente se, $C(\bar{I})=\Gamma_{+}(I)$.

Demonstração: Se $e(I)=n ! v(I)$, segue do Teorema 3.2 .6 e de $\Gamma_{+}(I)=\Gamma_{+}\left(I_{0}\right)$ que $e(I)=e\left(I_{0}\right)$. Pelo Teorema de Rees, temos que $\bar{I}=\overline{I_{0}}$. Assim,

$$
C(\bar{I})=C\left(\overline{I_{0}}\right)=\Gamma_{+}\left(I_{0}\right)=\Gamma_{+}(I)
$$

pois $I_{0}$ é monomial.

Para mostrar a recíproca, consideramos

$$
K_{I}=\left(\left\{x^{k}: x^{k} \in \bar{I}\right\}\right) \quad \text { e } \quad w(I)=v\left(K_{I}\right)=v\left(\mathbb{R}_{+}^{n} \backslash \Gamma_{+}\left(K_{I}\right)\right)
$$

Observemos que $\Gamma_{+}(I)=C(\bar{I})$. Decorre do Teorema 3.2 .6 a seguinte seqüência de desigualdades:

$$
n ! w(I)=n ! v\left(K_{I}\right)=e\left(K_{I}\right) \geq e(\bar{I})=e(I) \geq e\left(I_{0}\right)=n ! v\left(I_{0}\right)=n ! v(I)
$$

Assim, se $\Gamma_{+}(I)=C(\bar{I})$, então $v(I)=w(I)$ e, portanto, $e(I)=n ! v(I)$. 
Lema 3.3.11. Sejam I um ideal de codimensão finita em $\mathcal{O}_{n}, K_{I}=\left(\left\{x^{-k}: x^{k} \in \bar{I}\right\}\right)$ e $w(I)=v\left(K_{I}\right)$. Então, $e(I)=n ! w(I)$ se, e somente se, $C(\bar{I})=\Gamma_{+}(I)$.

Demonstração: De fato, se $e(I)=n ! w(I)$, então $e(\bar{I})=e(I)=n ! w(I)=e\left(K_{I}\right)$ pois $K_{I}$ é monomial. Assim, segue do teorema de Rees que $\bar{I}=\overline{K_{I}}$. Daí, $C(\bar{I})=\Gamma_{+}\left(K_{I}\right)=$ $\Gamma_{+}\left(\overline{K_{I}}\right)=\Gamma_{+}(\bar{I})=\Gamma_{+}(I)$. Reciprocamente, se $C(\bar{I})=\Gamma_{+}(I)$, então $w(I)=v\left(K_{I}\right)=$ $v\left(\mathbb{R}_{+}^{n} \backslash C(\bar{I})\right)=v(I)$. Segue da proposição anterior que $e(I)=n ! v(I)=n ! w(I)$.

As Proposições 3.3 .9 e 3.3.10 nos fornecem o seguinte

Teorema 3.3.12 (M. J. Saia, [14]). Seja I um ideal de codimensão finita em $\mathcal{O}_{n}$. Então, I é Newton não-degenerado se, e somente se, $C(\bar{I})=\Gamma_{+}(I)$.

Resumimos a caracterização que fizemos de ideal Newton não-degenerado no seguinte

Corolário 3.3.13. Seja I um ideal de codimensão finita em $\mathcal{O}_{n}$. As seguintes condições são equivalentes:

(i) I é Newton não-degenerado;

(ii) $e(I)=n ! v(I)$;

(iii) $e(I)=n ! w(i)$;

(iv) $\bar{I}$ é monomial;

(v) I admite uma redução Newton não-degenerada;

(vi) $I_{0} \subset \bar{I}$;

(vii) $\bar{I}=\left\{f \in \mathcal{O}_{n} ; \Gamma_{+}(f) \subset \Gamma_{+}(I)\right\}$.

A primeira demonstração do Teorema 3.3.12 foi obtida por M.J. Saia em [14], considerando a construção do mergulho toroidal associado ao poliedro de Newton $\Gamma_{+}(I)$. Observamos que em [15], ainda considerando a construção do mergulho toroidal associado a $\Gamma_{+}(I)$, M.J. Saia descreve um algoritmo para a determinação do poliedro $C(\bar{I})$ para ideais que são Newton degenerados mas, com codimensão finita em $\mathcal{O}_{n}$. 
A determinação do poliedro $\Gamma_{+}(I)$ em termos da multiplicidade $e(I)$ (e vice-versa) para ideais Newton degenerados não é conhecida. 


\section{Capítulo 4}

\section{Equisingularidade}

\subsection{Whitney equisingularidade}

No estudo de equisingularidades, deseja-se descobrir quando um conjunto $X$ algébrico sobre $\mathbb{R}$ ou $\mathbb{C}$, ou analítico, pode ser considerado equisingular ao longo de um subconjunto não singular $Y \subset X$ em um ponto $0 \in Y$.

Consideramos germes de hipersuperfícies analíticas complexas reduzidas $(X, 0) \subset$ $\left(\mathbb{C}^{n+1}, 0\right)$.

Dizemos que $X$ tem uma estratificação de Whitney, se existe uma partição de $X$ localmente finita em subespaços não singulares $Y_{\alpha}$ localmente fechados, com as seguintes propriedades:

(i) $\bar{Y}_{\alpha}$ e $\bar{Y}_{\alpha}-Y_{\alpha}$ são subespaços fechados de $X, \operatorname{dim}\left(\bar{Y}_{\alpha}-Y_{\alpha}\right)<\operatorname{dim} Y_{\alpha}$, e se $Y_{\alpha} \cap Y_{\beta} \neq \varnothing$ então $Y_{\beta} \subset \bar{Y}_{\alpha}$.

(ii) Se $Y_{\beta} \subset \overline{Y_{\alpha}}$, as seguintes condições de incidência acontecem:

Condições de Whitney

(a) Para qualquer seqüência de pontos $y_{i} \in Y_{\alpha}$ convergindo para um ponto $y \in Y_{\beta}$ e tal que as direções dos espaços tangentes $T_{Y_{\alpha}, y_{i}}$ convergem para um hiperplano $T$, temos: $T_{Y_{\beta}, y} \subset T$.

(b) Dadas sequiências de pontos $y_{i} \in Y_{\alpha}$ e $z_{i} \in Y_{\beta} \operatorname{com} y_{i} \rightarrow y$ e $z_{i} \rightarrow y$ e tais 
que $T_{Y_{\alpha}, y_{i}}$ converge para um plano $T$ e a direção da secante $\overline{z_{i} y_{i}} \in \mathbb{K}^{n+1}$ converge para $l$, temos $l \subset T$.

Se $y$ é fixado, diremos "condições de Whitney em $y$ ".

(iii) $\mathrm{O}$ conjunto $X^{0}$ dos pontos regulares de $X$ é um estrato (não é necessária a conexão para ser um estrato) e todos os outros estratos estão em seu fecho.

Definição 4.1.1. O par $(X, Y)$ é $W$-equisingular em $y=0$ se $Y$ é um estrato de alguma estratificação de Whitney de $X$, em uma vizinhança do zero.

A W-equisingularidade do par $(X, Y)$ implica na existência de uma vizinhança tubular $T_{Y}$ de $Y$, com retração $\Pi_{Y}: T_{Y} \rightarrow Y$ tal que $\Pi_{Y}:\left[T_{Y}, T_{Y} \cap \bar{X}, Y\right] \rightarrow Y$ é um fibrado localmente topologicamente trivial com fibras do tipo cone.

\subsubsection{Whitney equisingularidade e o fecho integral de ideais}

Seja $X_{G}=G^{-1}(0)$ uma hipersuperfície em $\mathbb{C}^{n} \times \mathbb{C}$, em que $G:\left(\mathbb{C}^{n} \times \mathbb{C}, 0\right) \rightarrow(\mathbb{C}, 0)$ é uma deformação a um parâmetro do germe $g$, ou seja, $G(x, 0)=g(x), \quad \forall \quad x$. O par $\left\{X_{G}, 0 \times \mathbb{C}\right\}$ é Whitney equisingular em 0 se $0 \times \mathbb{C}$ é um estrato de uma estratificação de $X_{G}$ em uma vizinhança de 0 .

B. Teissier, provou em [20] uma importante relação de dependência inteira na determinação das condições de Whitney.

Teorema 4.1.2 (A condição $(c)$ de Teissier). $O \operatorname{par}\left\{X_{G}, 0 \times \mathbb{C}\right\}$ é Whitney equisingular se, e somente se, $\frac{\partial G}{\partial t}$ pertence ao fecho integral do ideal em $\mathcal{O}_{n+1}$ gerado $\operatorname{por}\left\{x_{i} \frac{\partial G}{\partial x_{j}} \quad \forall \quad i, j \in\{1, \ldots, n\}\right\}$.

No que segue, para um germe $g:\left(\mathbb{C}^{n}, 0\right) \rightarrow(\mathbb{C}, 0)$ denotamos por $I$ o ideal $\left(x_{i} \frac{\partial g}{\partial x_{j}}, i, j=1, \ldots, n\right)$.

Definição 4.1.3. Dado um germe $g \in \mathcal{O}_{n}$, seu poliedro de equisingularidade, denotado por $\varepsilon(g)$, é definido como o fecho convexo do conjunto $\bigcup\left\{m+\mathbb{R}_{+}^{n}: x^{m} \in \bar{I}\right\}$. 
Uma questão natural é identificar todas as direções $\theta$ tais que $\Gamma_{+}(\theta) \subset \varepsilon(g)$ e dar condições necessárias e suficientes, em termos do poliedro $\varepsilon(g)$, para a Whitney equisingularidade do par $\left\{G^{-1}(0), 0 \times \mathbb{C}\right\} \operatorname{com} G(x, t)=g(x)+t \theta$.

Uma condição necessária para que o monômio $x^{m} \in \overline{\left(x_{i} \frac{\partial G}{\partial x_{j}}\right)}$ e, portanto, para a Whitney equisingularidade do $\operatorname{par}\left\{X_{G}, 0 \times \mathbb{C}\right\}$, é que $m \in \varepsilon(g)$. Isso é o que diz o próximo lema.

Lema 4.1.4. Seja $G(x, t)=g(x)+t x^{m}$ uma deformação de um germe $g$ com singularidade isolada na origem. Uma condição necessária para $x^{m} \in \overline{\left(x_{i} \frac{\partial G}{\partial x_{j}}\right)}$ em $\mathcal{O}_{n+1}$ é $m \in \varepsilon(g) e m \mathbb{R}^{n}$.

Demonstração: Se $m \notin \varepsilon(g)$ em $\mathbb{R}^{n}$, segue do critério avaliativo do Teorema 2.3.6 que existe uma curva $\phi:(\mathbb{C}, 0) \rightarrow\left(\mathbb{C}^{n}, 0\right)$ tal que

$$
f i l_{\mathcal{O}_{1}}\left(x^{m} \circ \phi\right)<f i l_{\mathcal{O}_{1}}\left(x_{i} \frac{\partial g}{\partial x_{j}} \circ \phi\right) \quad \forall i, j=1, \ldots, n ;
$$

em que $f i l_{\mathcal{O}_{1}}\left(\sum a_{l} t^{l}\right)=\min \left\{l: a_{l} \neq 0\right\}$ para qualquer série de Taylor $\sum a_{l} t^{l} \in \mathcal{O}_{1}$.

Considere $\psi$ uma curva em $\mathcal{O}_{n+1}$ definida por $\psi(\lambda)=(\phi(\lambda), 0)$. Como

$$
x_{i} \frac{\partial G}{\partial x_{j}}=x_{i} \frac{\partial g}{\partial x_{j}}+t x_{i} \frac{\partial x^{m}}{\partial x_{j}}
$$

para todo $i, j=1, \ldots, n$, temos que $f i l_{\mathcal{O}_{1}}\left(x_{i} \frac{\partial g}{\partial x_{j}} \circ \phi\right)=f i l_{\mathcal{O}_{1}}\left(x_{i} \frac{\partial G}{\partial x_{j}} \circ \psi\right)$. Assim, o resultado segue do Teorema 2.3.6.

O lema a seguir, mostra que o poliedro de equisingularidade nos dá ainda, uma condição suficiente para a Whitney equisingularidade do $\operatorname{par}\left\{G^{-1}(0), 0 \times \mathbb{C}\right\}$.

Lema 4.1.5. Seja $G(x, t)=g(x)+t x^{m},|t| \leq 1$, uma deformação de um germe $g$. Se $x_{i} \frac{\partial x^{m}}{\partial x_{j}} \in \bar{I}$ para todo $i, j \in\{1, \ldots, n\}$, então $x^{m} \in \overline{\left(x_{i} \frac{\partial G}{\partial x_{j}}\right)}$.

Demonstração: Segue da hipótese, que existe uma constante $0<\epsilon<1$ tal que

$$
|t|\left|x_{i} \frac{\partial x^{m}}{\partial x_{j}}\right| \leq \epsilon \sup _{i, j}\left|x_{i} \frac{\partial g}{\partial x_{j}}(x)\right|
$$

para todo $(x, t)$ em uma vizinhnça $U$ de $(0,0)$ em $\mathbb{C}^{n} \times \mathbb{C}$. Portanto,

$$
\sup _{i, j}\left|x_{i} \frac{\partial G}{\partial x_{j}}(x)\right|=\sup _{i, j}\left|x_{i} \frac{\partial g}{\partial x_{j}}(x)+t x_{i} \frac{\partial x^{m}}{\partial x_{j}}(x)\right|
$$




$$
\begin{aligned}
& \geq \sup _{i, j}\left|x_{i} \frac{\partial g}{\partial x_{j}}(x)\right|-|t| \sup _{i, j}\left|x_{i} \frac{\partial x^{m}}{\partial x_{j}}(x)\right| \\
& \geq(1-\epsilon) \sup _{i, j}\left|x_{i} \frac{\partial g}{\partial x_{j}}(x)\right| .
\end{aligned}
$$

Segue da condição de crescimento do Teorema 2.3.6 que existe uma contante $k>0$ tal que

$$
\left|x^{m}\right| \leq k \sup _{i, j}\left|x_{i} \frac{\partial g}{\partial x_{j}}(x)\right|
$$

para todo $x$ em uma vizinhança $V$ de 0 em $\mathbb{C}^{n}$. Assim,

$$
\left|x^{m}\right| \leq k \sup _{i, j}\left|x_{i} \frac{\partial g}{\partial x_{j}}(x)\right| \leq \frac{k}{1-\epsilon} \sup _{i, j} x_{i}\left|x_{i} \frac{\partial G}{\partial x_{j}}(x)\right|
$$

para todo $x$ na vizinhança $(V \times \mathbb{C}) \cap U$ de $(0,0)$ em $\mathbb{C}^{n} \times \mathbb{C}$.

Observação 4.1.6. A técnica usada na demonstração do lema acima, como também na Proposição 4.2.7 foi inicialmente usada por T.C.Kuo em [10] e por E. Yoshinaga no Lema 2.1 em [24].

Nosso objetivo agora, é caracterizar o poliedro de equisingularidade $\varepsilon(g)$ em termos do poliedro de Newton $\Gamma_{+}(I)$ do ideal $I$. Quando $\Gamma_{+}(I)$ é Newton não-degenerado, o Lema 3.3.3 e o Teorema 3.3.12 nos garantem a seguinte

Proposição 4.1.7. Dado um germe $g:\left(\mathbb{C}^{n}, 0\right) \rightarrow(\mathbb{C}, 0)$ em $\mathcal{O}_{n}$, temos:

(i) $\varepsilon(g) \subset \Gamma_{+}(I)$

(ii) $\varepsilon(g)=\Gamma_{+}(I)$ se, e somente se, $\Gamma_{+}(I)$ é nâo degenerado.

\subsection{Trivialidade topológica}

Uma família $F:\left(\mathbb{C}^{n} \times \mathbb{C}, 0\right) \rightarrow(\mathbb{C})$ tal que $F(0, t)=0, \quad \forall t \in \mathbb{C}$, é topologicamente trivial se existem um $t_{0} \in \mathbb{C}$ e um homeomeorfismo $H:\left(\mathbb{C}^{n} \times \mathbb{C}, 0\right) \rightarrow$ $\left(\mathbb{C}^{n} \times \mathbb{C}, 0\right)$ do tipo $H(x, t)=(h(x, t), t)$ tal que $(F \circ H)(x, t)=F\left(x, t_{0}\right)$ para todo $(x, t) \in \mathbb{C}^{n} \times \mathbb{C}$. Sem perda de generalidade, supomos que $t_{0}=0$.

Seja $F:\left(\mathbb{C}^{n} \times[0, \epsilon), 0\right) \rightarrow(\mathbb{C}, 0) \operatorname{com} \epsilon>0$, uma deformação a um parâmetro de um germe $f:\left(\mathbb{C}^{n}, 0\right) \rightarrow(\mathbb{C}, 0)$. Se a variedade $X_{F}=F^{-1}(0)$ é Whitney equisingular 
ao longo do espaço de parâmetros em 0 , então a família é topologicamente trivial. A recíproca não vale como mostra o seguinte exemplo de Briançon-Speder.

Exemplo 4.2.1. A familia de hipersuperfícies em $X_{t} \in \mathbb{C}^{3}$ definida pelas equações

$$
F_{t}\left(x_{1}, x_{2}, x_{3}\right)=F\left(x_{1}, x_{2}, x_{3}, t\right)=x_{3}^{5}+x_{2}^{7} x_{1}+x_{1}^{15}+t x_{2}^{6} x_{3}=0
$$

tem tipo topológico constante, logo é topologicamente trivial. No entanto, a variedade $F^{-1}(0) \in \mathbb{C}^{4}$ não é Whitney equisingular ao longo do espaço de parâmetros em 0 /ver [20], pag 599].

Se uma família $F(x, t)=F_{t}(x)$ é topologicamente trivial e possui singularidade isolada, então é $\mu$-constante. Ou seja, temos $\mu\left(F_{t}\right)=\mu\left(F_{0}\right)$ para valores pequenos de $t$.

\subsubsection{Trivialidade topológica e fecho integral}

Sejam $f \in \mathcal{O}_{n}$ e $F:\left(\mathbb{C}^{n} \times \mathbb{C}, 0\right) \rightarrow(\mathbb{C})$ uma deformação a um parâmetro de $f$; isto é, $F(x, 0)=f(x) \quad \forall x \in \mathbb{C}^{n}$. Apresentamos a seguir, um resultado que relacionam as deformações $\mu$ constantes de $f$ e o fecho integral do ideal jacobiano $J(F)=\left(\frac{\partial F}{\partial x_{1}}, \ldots, \frac{\partial F}{\partial x_{n}}\right)$ de $F$ com respeito às coordenadas $x$.

Definição 4.2.2. Um elemento $h \in \mathcal{O}_{n}$ pertence ao fecho integral estrito do ideal I de $\mathcal{O}_{n}$, denotado por $\bar{I}^{+}$se para cada sistema de geradores $h_{1}, \ldots, h_{k}$ de $I, f i l_{\mathcal{O}_{1}}(h \circ \varphi)>$ $\inf \left\{f_{i l_{\mathcal{O}_{1}}}\left(h_{1} \circ \varphi\right), \ldots\right.$, fil $\left._{\mathcal{O}_{1}}\left(h_{k} \circ \varphi\right)\right\}$ para cada curva analítica $\varphi:(\mathbb{C}, 0) \rightarrow\left(\mathbb{C}^{n}, 0\right)$.

Teorema 4.2.3 (Greuel [6], pag. 161). Seja $F:\left(\mathbb{C}^{n} \times[0, \epsilon), 0\right) \rightarrow(\mathbb{C}, 0)$ uma deformação a um parâmetro de um germe holomorfo $f ;\left(\mathbb{C}^{n}, 0\right) \rightarrow(\mathbb{C}, 0)$ com singularidade isolada. As seguintes condições são equivalentes:

(i) F é topologicamente trivial,

(ii) $\frac{\partial F}{\partial t} \in \overline{J(F)}^{+}$,

(iii) $\frac{\partial F}{\partial t} \in \overline{J(F)}$, 
(iv) $\frac{\partial F}{\partial t} \in \sqrt{J(F)}$

(v) A curva polar de $F$ com respeito a $\{t=0\}$ não se fatora, isto é, $\left\{(x, t) \in \mathbb{C}^{n} \times \mathbb{C}: \frac{\partial F}{\partial x_{i}}(x, t)=0, \quad \forall i=1, \ldots, n\right\}=\{0\} \times \mathbb{C}$ próximo de $(0,0)$.

\subsubsection{Trivialidade topológica e poliedro de Newton}

Dado um germe $f \in \mathcal{O}_{n}$, denotamos por $J(f)$ o ideal jacobiano de $f$, ou seja, o ideal gerado pelo sistema $\left\{\frac{\partial f}{\partial x_{1}}, \ldots, \frac{\partial f}{\partial x_{n}}\right\}$. Usamos o fecho integral deste ideal para encontrar condições para a $\mu$-constância de uma deformação de $f$.

Definição 4.2.4. Denotamos por $T(f)$ o fecho convexo em $\mathbb{R}_{+}^{n}$ do conjunto

$$
\left\{m+\mathbb{R}_{+}^{n}: x^{m} \in \overline{J(f)}\right\} .
$$

Lema 4.2.5. Seja $F(x, t)=f(x)+t g(x)$ uma deformação de primeira ordem de $u m$ germe complexo $f$ com singularidade isolada. Se a familia $f_{t}$ é topologicamente trivial, então $\Gamma_{+}(g) \subseteq T(f)$.

Demonstração: Se $F$ é $\mu$-constante, então segue do Teorema 4.2 .3 que $g(x)=$

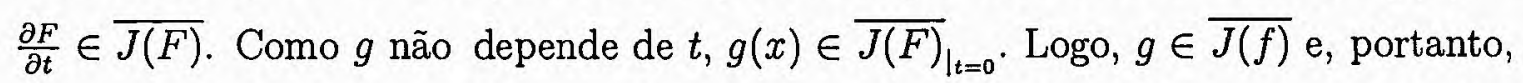
$\Gamma_{+}(g) \subseteq T(f)$.

Descrevemos agora, condições suficientes para a trivialidade topológica. Yoshinaga fornece em [24] condições para a trivialidade topologica de famílias do tipo $F(x, t)=f(x)+t g(x)$ em termos do poliedro gradiente $\Lambda_{+}(f)$, definido como o fecho convexo do conjunto

$$
\bigcup\left\{m+\mathbb{R}_{+}^{n}:\left|x_{1} \frac{\partial f}{\partial x_{1}}\right|+\cdots+\left|x_{n} \frac{\partial f}{\partial x_{n}}\right| \geq \varepsilon\left|x^{m}\right|\right\},
$$

para um $\varepsilon(m)$ positivo em uma vizinhança da origem em $\mathbb{C}^{n}$. Ainda neste trabalho, Yoshinaga mostra que se $\Gamma_{+}(g) \subset \Lambda_{+}(f)$, então $F(x, t)=f(x)+t g(x)$ é topologicamente trivial para valores suficientemente pequenos de $t$. Damon-Gaffney em [4], também apresentam resultados similares para a trivialidade topológica. 
Dizer que um germe $g$ satisfaz a condição $\Gamma_{+}(g) \subset \Lambda_{+}(f)$ é equivalente a $g$ pertencer ao fecho integral do ideal gerado pelo sistema $\left\{x_{1} \frac{\partial f}{\partial x_{1}}, \ldots, x_{n} \frac{\partial f}{\partial x_{n}}\right\}$. Como conseqüência dos resultados de Yoshinaga e Damon-Gaffney, temos a seguinte

Proposição 4.2.6. Seja $F(x, t)=f(x)+\delta(t) g(x)$ uma deformação de $f$ com singularidade isolada em 0 . Se $\Gamma_{+}(g) \subset \Lambda_{+}(f)$, então $F(x, t)$ é topologicamente trivial para valores suficientemente pequenos de $t$.

Apresentamos a seguir, uma condição suficiente para a $\mu$-constância em termos do poliedro $T(f)$.

Proposição 4.2.7. Seja $F(x, t)=f(x)+\delta(t) g(x)$ uma deformação de um germe complexo $f$ com singularidade isolada. Se $\Gamma_{+}(J(g)) \subseteq T(f)$, entãa $F(x, t)$ é topologicamente trivial para valores pequenos de $t$.

Demonstração: Se $\Gamma_{+}(J(g)) \subseteq T(f)$, então segue do Teorema 2.3.6 que para cada $i \in\{1, \ldots n\}$ existem vizinhanças $U_{i}$ de 0 e constantes $C_{i}>0$ tais que

$$
\left|\frac{\partial g}{\partial x_{i}}\right| \leq C_{i} \sup \left\{\left|\frac{\partial f}{\partial x_{1}}\right|, \ldots,\left|\frac{\partial f}{\partial x_{n}}\right|\right\} \text {. }
$$

Daí,

$$
|\delta(t)|\left|\frac{\partial g}{\partial x_{i}}\right| \leq|\delta(t)| C_{i} \sup \left\{\left|\frac{\partial f}{\partial x_{1}}\right|, \ldots,\left|\frac{\partial f}{\partial x_{n}}\right|\right\} .
$$

Assim, para uma vizinhança $U \subset U_{i} \quad \forall i=1, \ldots, n$, temos

$$
\begin{aligned}
\sup _{i}\left|\frac{\partial F}{\partial x_{i}}\right| & =\sup _{i}\left|\frac{\partial f}{\partial x_{i}}+\delta(t) \frac{\partial g}{\partial x_{i}}\right| \\
& \geq \sup _{i}\left|\frac{\partial f}{\partial x_{i}}\right|-\sup _{i}|\delta(t)|\left|\frac{\partial g}{\partial x_{i}}\right| \\
& \geq \sup _{i}\left|\frac{\partial f}{\partial x_{i}}\right|-|\delta(t)| C_{i} \sup _{i}\left\{\left|\frac{\partial f}{\partial x_{i}}\right|\right\} \\
& \geq\left(1-|\delta(t)| C_{i}\right) \sup _{i}\left|\frac{\partial f}{\partial x_{i}}\right| \\
& \geq(1-\alpha) \sup _{i}\left|\frac{\partial f}{\partial x_{i}}\right|,
\end{aligned}
$$

para algum $0<\alpha<1 \operatorname{com}\left|\delta(t) C_{i}\right| \leq \alpha \quad \forall \quad i=1, \ldots, n$.

Esta desigualdade implica que $\overline{\left(\frac{\partial f}{\partial x_{1}}, \ldots, \frac{\partial f}{\partial x_{n}}\right)} \subset \overline{\left(\frac{\partial F}{\partial x_{1}}, \ldots, \frac{\partial F}{\partial x_{n}}\right)}$. 
Agora, mostramos que para cada curva analítica $\psi:(\mathbb{C}, 0) \rightarrow\left(\mathbb{C}^{n+1}, 0\right)$,

$$
f i l_{\mathcal{O}_{1}}\left(\frac{\partial F}{\partial t} \circ \psi\right) \geq \min _{i}\left\{f i l_{\mathcal{O}_{1}}\left(\frac{\partial F}{\partial x_{i}} \circ \psi\right)\right\}
$$

Escrevendo $\psi=(\varphi, \lambda)$, temos

$$
f i l_{\mathcal{O}_{1}}\left(\frac{\partial F}{\partial t} \circ \psi\right) \geq \min _{i}\left\{f i l_{\mathcal{O}_{1}}\left(\delta^{\prime} \circ \lambda\right)+f i l_{\mathcal{O}_{1}}(g \circ \varphi)\right\} \geq \min _{s}\left\{1+f i l_{\mathcal{O}_{1}}(g \circ \varphi)\right\} .
$$

Segue da hipótese que $g \in \overline{\left(\frac{\partial f}{\partial x_{1}}, \ldots, \frac{\partial f}{\partial x_{n}}\right)}$, portanto,

$$
f i l_{\mathcal{O}_{1}}(g \circ \varphi) \geq \min _{i}\left\{f i l_{\mathcal{O}_{1}}\left(\frac{\partial f}{\partial x_{i}} \circ \varphi\right)\right\} \geq \min _{i}\left\{f i l_{\mathcal{O}_{1}}\left(\frac{\partial F}{\partial x_{i}} \circ \psi\right)\right\} .
$$

Assim, $f i l_{\mathcal{O}_{1}}\left(\frac{\partial F}{\partial t} \circ \psi\right) \geq \min _{i}\left\{1+f i l_{\mathcal{O}_{1}}(g \circ \varphi)\right\} \geq \min \left\{f i l_{\mathcal{O}_{1}}\left(\frac{\partial F}{\partial x_{i}} \circ \psi\right)\right\}$ e o resultado segue do Teorema 4.2.3. 


\section{Referências Bibliográficas}

[1] - C.B. Ausina, Singularidades de Thom-Boardman en Deformaciones Genéricas de Gérmenes de Aplicaciones y Métodos para el Cálculo de Clausuras Integrales de Ideales, Tesis Doctoral, Departament de Geometria i Topologia, Universitat de Valência, (2000).

[2] - N. Bourbaki, Elements of Mathematics, Commutative Algebra, Addison-Wesley Publishing Company, (1972).

[3] - E. Brieskorn \& H. Knörrer, Plane Algebraic Curves, Birkhäuser, (1986).

[4] - J.N. Damon \& T. Gaffney, Topological Triviality of Deformations of Functions and Newton Filtrations, Inv. Math., 72, (1983), 335-358.

[5] - D. Eisenbud, Commutative Algebra with a View Toward Algebraic Geometry, Graduate Texts in Mathematics, vol. 50, Springer (1996).

[6] - G.M. Greuel, Constant Milnor Number Implie Constant Multiplicity for Quasihomogeneous Singularities, Manuscripta Math. 56, (1986), 159-166.

[7] - R. Hartshorne, Algebraic Geometry, Gaduate Texts in Mathematics, vol 52, Springer-Verlag, (1987)

[8] - H. Hironaka, Resolution of Singularities of an Algebraic Variety over a Field of Characteristic Zero, I, II, Annals of Math. 79, (1964), 109-203.

[9] - G.R. Kempf, Algebraic Varieties, London Mathematical Society Lectures Notes Séries, 172, Cambridge University Press, (1993). 
[10] - T.C. Kuo, On $C^{0}$-Sufficiency of Jets of Potential Function, Topology, 8, (1969), 167-171.

[11] - A.G. Kouchnirenko, Polyèdres de Newton et Nombres de Milnor, Invent. Math., 32 (1976), 1-31.

[12] - H. Matsumura, Commutative Ring Theory, Cambridge Studies in Advanced Mathematics, 8, (1997).

[13] - D. Mumford The Red Book of Varieties and Schemes, 2nd edition, Lectures Notes in Mathematics, 1358, Springer, (1999).

[14] - M.J. Saia, The Integral Closure of Ideals and the Newton Filtration, Journal of Algebraic Geometry, 5 (1996), 1-11.

[15] - M.J. Saia The Integral Closure of Ideals and Whitney Equisingularity of Germs of Hypersurfaces, Matemática Contemporânea, 129, (1997), 183-198.

[16] - M.J. Saia \& J.N. Tomazela, Deformations with Constant Milnor Number and Multiplicity of Non-Degenerate Complex Hypersurfaces, Notas ICMC-USP, Série Matemática, 129, (2001).

[17] - M.J. Saia, T. Fukui, \& C. Bivia-Ausina Saia, M. J., Newton Filtrations, Graded Algebras and Codimension of Ideals, A ser publicado por: Math. Proc. of the Cambridge Phil. Soc., Vol. 133, part 1, Julho de 2002.

[18] - B. Teissier \& M. Lejeune, Closure Integrale des Ideaux et Equisingularite, Séminaire Centre de Mathématiques Ecole Polytechnique, (1974).

[19] - B. Teissier, Monômes, volumes et multiplicités, Introduction à la théorie des singularités II, Travaux en Cours 37 (1998), 127-141.

[20] - B. Teissier, Introduction to Equisingularity Problems, Algebraic Geometry, Proceedings of Symposia in Pure Mathematics 29, Amer. Math. Soc., Providence, RI, (1975), 593-632. 
[21] - B. Teissier, Variétes Polaires II. Multiplicités Polaires, Section Planes et Conditions de Whitney, Lectures Notes in Mathematics, 961, (1982), 314-491.

[22] - B. Teissier, Cycles É vanescents, Sections Planes et Conditions de Whitney in Singularités à Cargèse, (1972), Astérisque, 7-8, (1983).

[23] - H. Whitney, Complex Analytic Varieties, Addison-Wesley Publishing Company, (1972).

[24] - E. Yoshinaga, Topological Principal Part of Analytic Functions, Trans. of the A.M.S. 314, 2, (1989), 803-813. 